\title{
A Survey and Comparative Study of Broadcast Warning Message Dissemination Schemes for VANETs
}

\author{
Julio A. Sanguesa, ${ }^{1}$ Manuel Fogue, ${ }^{1}$ Piedad Garrido, ${ }^{1}$ Francisco J. Martinez, \\ Juan-Carlos Cano, ${ }^{2}$ and Carlos T. Calafate ${ }^{2}$ \\ ${ }^{1}$ Computer Science and System Engineering Department, University of Zaragoza, C/Atarazana 2, 44003 Teruel, Spain \\ ${ }^{2}$ Computer Engineering Department, Universitat Politècnica de València, Camino de Vera, s/n, 46022 Valencia, Spain
}

Correspondence should be addressed to Francisco J. Martinez; f.martinez@unizar.es

Received 20 November 2015; Accepted 15 March 2016

Academic Editor: Massimo Merro

Copyright (C) 2016 Julio A. Sanguesa et al. This is an open access article distributed under the Creative Commons Attribution License, which permits unrestricted use, distribution, and reproduction in any medium, provided the original work is properly cited.

\begin{abstract}
Vehicle-to-vehicle (V2V) communications also known as vehicular ad hoc networks (VANETs) allow vehicles to cooperate to increase driving efficiency and safety on the roads. In particular, they are forecasted as one of the key technologies to increase traffic safety by providing useful traffic services. In this scope, vehicle-to-vehicle dissemination of warning messages to alert nearby vehicles is one of the most significant and representative solutions. The main goal of the different dissemination strategies available is to reduce the message delivery latency of such information while ensuring the correct reception of warning messages in the vehicle's neighborhood as soon as a dangerous situation occurs. Despite the fact that several dissemination schemes have been proposed so far, their evaluation has been done under different conditions, using different simulators, making it difficult to determine the optimal dissemination scheme for each particular scenario. In this paper, besides reviewing the most relevant broadcast dissemination schemes available in the recent literature, we also provide a fair comparative analysis by evaluating them under the same environmental conditions, focusing on the same metrics, and using the same simulation platform. Overall, we provide researchers with a clear guideline of the benefits and drawbacks associated with each scheme.
\end{abstract}

\section{Introduction}

In the past, the efforts of administrations to increase traffic safety were focused on building more efficient and safer roads. Over the years, these efforts shifted to the pursuit of creating faster cars to overcome longer distances, thus focusing on mechanical and automotive engineering. Afterward, car manufacturing was greatly impacted by electronics technology, and so sensors and Electronic Control Units (ECUs) were installed on vehicles to make them more sensitive and intelligent and basically safer to drive on [1]. Nowadays, innovations achieved in the field of networking technologies and particularly wireless mobile communications are being integrated into vehicles and roads. This impact will exceptionally modify how people will drive in the future and how transportation systems will be perceived. In particular, a revolution over the next decade is expected, creating a major social, economic, and global impact.
Vehicular communications should not be considered as mere basic data transfers since new opportunities to improve road safety and comfort are also available. The applications and potential advantages of vehicular communications, especially those able to enhance driving efficiency and road safety, are diverse. In fact, the interest in this area has grown considerably, receiving a noticeable attention from the research community during past years $[2,3]$.

The excitement about vehicular networks is mostly due to their wide range of solutions and open challenges. There are some important technical challenges to overcome, such as dissemination among vehicles, data delivery, high mobility and speeds of communicating vehicles, or real-time requirements. Such challenges and opportunities justify the increasing interest in vehicular networks of carmakers, governments, industries, and academia [4].

In this work, we present a survey and tutorial of the most relevant broadcast dissemination schemes proposed 
for vehicular environments so far. Specifically, we review and classify twenty-three different dissemination schemes which have been proposed. All these approaches try to improve the alert dissemination process, while mitigating the broadcast storm problem, that is, packet collisions caused by simultaneous broadcasting and packet distribution reduction due to severe message repetitions [5]. For the sake of clarity, the abbreviations used along this paper are presented at the end of the paper.

In modern Intelligent Transportation Systems, vehicles will be capable of automatically detecting dangerous situations, that is, their On-Board Units (OBUs), using the data gathered by the accelerometers and the rest of sensors available in the vehicle will be able to determine whether an accident has occurred [6]. Once the accident is detected, the vehicles will immediately send warning messages to their neighbors, and these messages will also be rebroadcasted by receiving vehicles to warn other vehicles, thereby preventing additional risks. More specifically, after a collision is detected, the OBU will build a warning message using the data gathered by the sensors available in the vehicle. All this information will also be useful to make a preliminary assessment of the accident severity [7] and the human and material resources required to optimize the rescue process, thus improving the assistance quality [8]. Therefore, an efficient warning message dissemination protocol should account for the most appropriate forwarding node for each message, thus maximizing the number of vehicles informed about the dangerous situation, while simultaneously reducing the time required to inform them and the amount of traffic generated in the wireless channel.

The rest of the paper is structured as follows: Section 2 presents some of the existing surveys that are closely related to this paper. Section 3 provides an introduction to vehicular networks, with an emphasis on vehicular ad hoc networks (VANETs). Section 4 reviews existing dissemination schemes including one-hop and multihop approaches. Moreover, we present a classification of existing proposals according to the characteristics and techniques adopted for the dissemination process. In Section 5 we detail the different simulation configurations and parameters used to assess existing broadcast dissemination schemes. Section 6 shows our simulation results, which have been performed under the same conditions, presenting and discussing the advantages and drawbacks of each proposed technique. Derived from simulation results and a qualitative analysis, in Section 7 we summarize the lessons learned, providing some considerations for future research. Lastly, Section 8 closes this paper.

\section{Existing VANET-Related Surveys}

Although some works (e.g., [9]) have surveyed existing broadcast protocols for mobile ad hoc networks (MANETs), to the best of our knowledge there are no specific VANEToriented works offering an overview of recent dissemination approaches.

In fact, despite the importance of warning message dissemination schemes in ITS safety applications, there is no survey so far that clearly presents and discusses the most relevant approaches proposed regarding warning message dissemination in VANETs. Additionally, existing proposals are usually evaluated under different conditions, making it quite difficult to determine what is the best dissemination scheme for each specific scenario. Below, we introduce some of the most relevant VANET-related surveys available.

Cheng et al. [10] presented VANET data dissemination results by structuring surveyed techniques into three categories: unicast, multicast, and geocast/broadcast techniques, describing the most important ideas in each category. They also considered location services and security issues, in the context of data dissemination in VANETs. Unlike our work, authors did not provide any comparative analysis in terms of dissemination performance of the different approaches studied.

Panichpapiboon and Pattara-Atikom [11] classified and provided an in-depth review of existing broadcasting protocols for VANETs. Despite the quality of this work, authors did not provide a thorough analysis of the characteristics of the protocols studied, nor was a fair comparison done. In particular, we consider carrying out an unbiased comparison essential, that is, under the same simulation environment, thereby providing researchers clear guidelines to accurately assess their proposals.

$\mathrm{X}$. Li and $\mathrm{H}$. Li [12] presented the most representative results of data dissemination in vehicle-to-vehicle (V2V) communications. In particular, their review was divided into three sections: routing protocols, mobility model, and security issues.

Regarding VANET mobility models, Harri et al. [13] presented a procedure for the implementation of vehicular mobility models. In addition, they introduced the different existing approaches for vehicular mobility and their relationship with network simulators. They also proposed a taxonomy of some existing mobility models commonly used when simulating vehicular ad hoc networks.

More recently, Jia et al. [14] presented a comprehensive study of platoon-based vehicular cyber-physical systems (VCPS). They also introduced two primary approaches based on VCPS, that is, the traffic dynamics, as well as the vehicular networking architecture and standards.

Although several authors have published surveys focused on different issues related to vehicular networks such as mobility models $[13,15]$, security attacks [16], revocation [17], or routing [18-20], none of these works specifically focused on the warning message dissemination process, nor on the broadcast schemes used when dangerous situations take place.

Moreover, existing works usually assess their proposals in very specific scenarios, with different vehicles densities, and under a wide variety of simulation tools. Therefore, unlike other surveys, in this work we assess the behavior of the most relevant existing broadcast dissemination protocols, evaluating them fairly, that is, under the same conditions, under same network model, and under same simulation tool and using the same performance metrics. We consider that such a fair evaluation is able to shed some light on the advantages and drawbacks of each solution, making it 


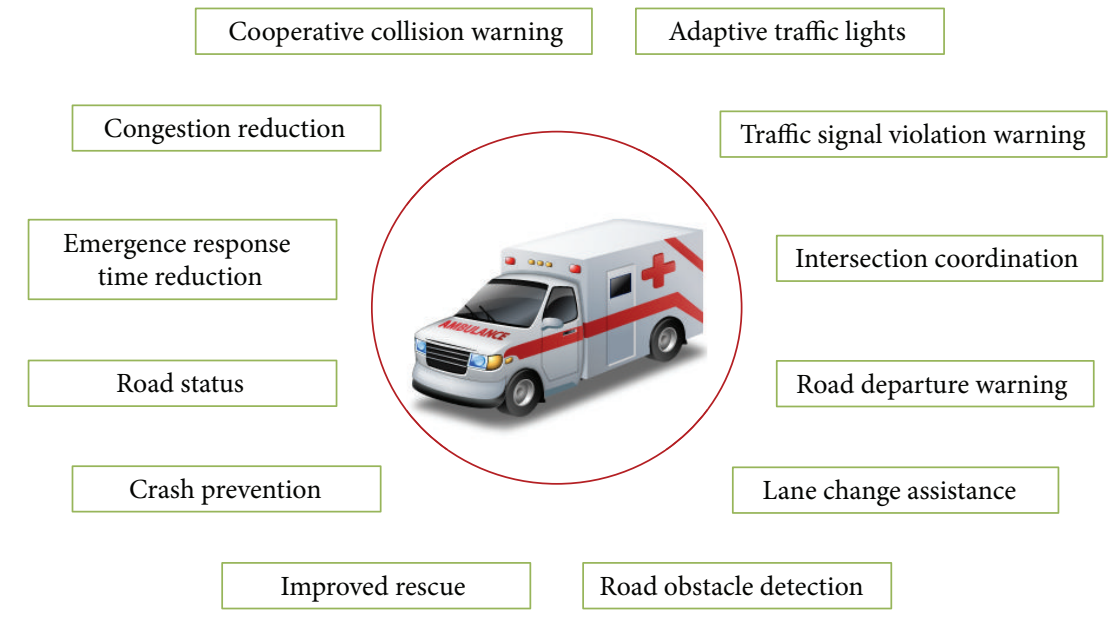

FIGURE 1: Traffic safety applications of vehicular networks.

possible to determine which one is the most suitable scheme to be used on each particular scenario.

\section{Vehicular Networks}

Vehicular networking is currently a challenging technology suitable for developing different types of applications related to efficient driving, smart vehicles, passengers' comfort, infotainment, and so forth. More specifically, vehicular networks (VNs) are wireless communication networks able to support enhanced driving and communications among vehicles. Accordingly, vehicles are able to communicate, thus creating dynamic wireless networks with other nearby vehicles and the infrastructure [21]. In particular, VNs include vehicle-toinfrastructure (V2I) [22] and vehicle-to-vehicle (V2V) [23] communications.

The specific characteristics of VNs promote the implementation of stimulating services and applications [24-26]. Next, we will introduce them in detail.

3.1. Applications of VNs. Applications of vehicular networks can be sorted into two main groups:

(i) Safety applications (see Figure 1) that attempt to improve passengers' safety by sending relevant information via V2V and V2I communications: this information can directly activate any automatic safety system or be simply provided to the driver. The proper operation of this kind of applications will only be possible once the penetration rate of communicationenabled vehicles is high enough.

(ii) Comfort and commercial applications (see Figure 2) that are aimed at improving traffic performance and increasing passengers' comfort: these applications usually involve routes optimization and $\mathrm{CO}_{2}$ emissions reduction or provide support for commercial transactions. Comfort and commercial applications must avoid interfering with safety applications [27].
3.2. Vehicular Ad Hoc Networks. Vehicular ad hoc networks (VANETs) are a particular subclass of vehicular networks (VNs) which represent a set of equipped vehicles communicating with each other wirelessly, without requiring the use of any infrastructure (see Figure 3).

A plethora of applications can be implemented in VANETs, including alert dissemination (to inform drivers about dangerous situations), collision avoidance and safety improvements (where communications can improve the driver's responsiveness), and real-time monitoring of traffic conditions (to reduce traffic congestion). Although VANETs seem to be mostly focused on enhancing traffic safety, they can also provide comfort applications between vehicles [29].

In VANETs, vehicles can access to Global Positioning Systems (GPS) and are provided with sensors able to gather location information (i.e., position, speed, direction, and acceleration). This information can also be broadcasted to its neighbors, enabling cooperative driving (e.g., neighboring vehicles can anticipate or evade potential risks).

Regarding safety, efficient warning message dissemination schemes are required since the main target is to decrease the latency of such critical data while ensuring the correct reception of alert information by neighbors [30]. When a vehicle detects an abnormal circumstance (e.g., roadworks, accidents, and bad weather), it immediately broadcasts the incident to neighboring vehicles, thus rapidly spreading the information to alert nearby vehicles. In all this process, the selected dissemination scheme is of utmost importance.

\section{Existing Broadcast Message Dissemination Schemes}

As previously mentioned, VANETs present some particular characteristics, such as organized networks and distribution of the processing tasks, a large amount of nodes (i.e., vehicles) moving at high speeds, a topology with high variability but constrained at the same time, varying mobility patterns and communication situations, and wireless signal blockage due 


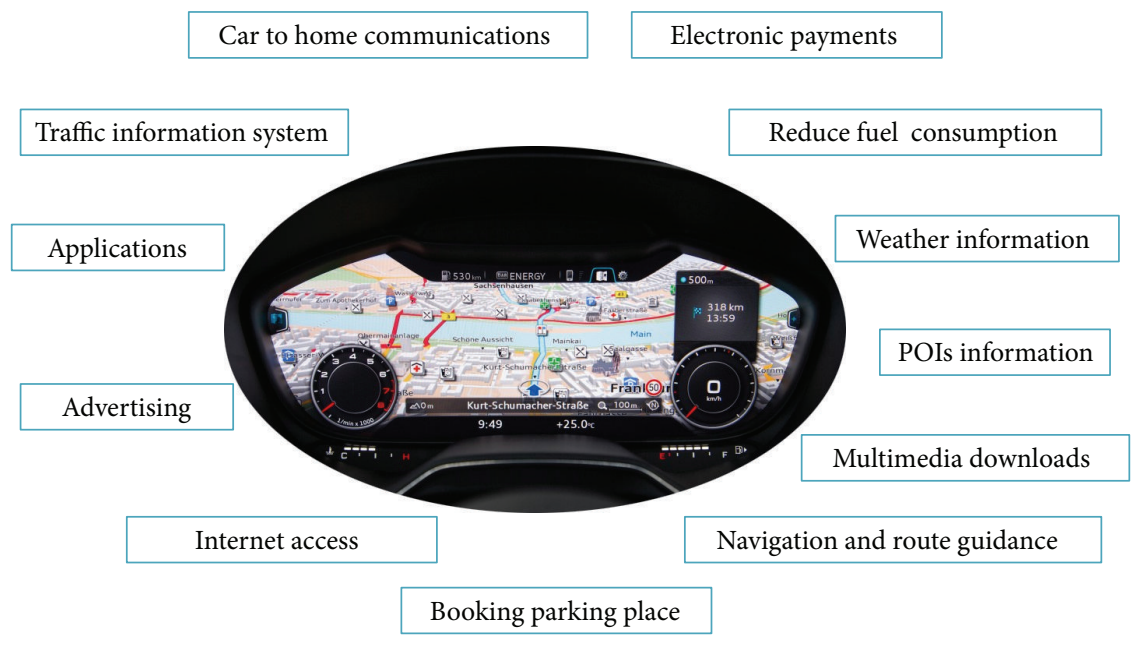

FIGURE 2: Comfort and commercial applications of vehicular networks.

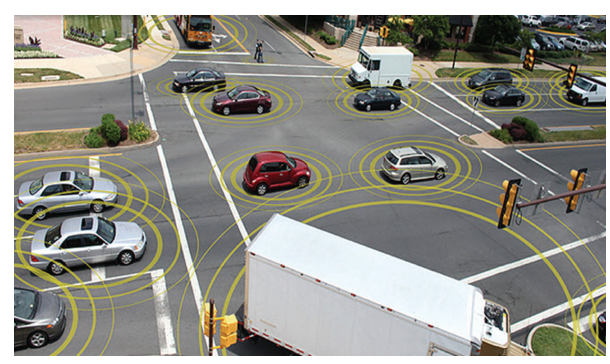

Figure 3: Typical VANET scenario [28].

to some obstacle (usually buildings), as well as network partitioning as a result of vehicle mobility. Under these conditions and with the objective of improving the dissemination process, several dissemination schemes have been proposed for vehicular environments.

Some existing works apply delay-tolerant networks to vehicular networks [31, 32]. The goal of these schemes is to allow communication between different clusters of vehicles, especially in sparse environments [33]. However, they usually require more resources, and their utility is very limited in warning message dissemination scenarios, where notification time is a critical factor. The long delay allowed in these networks in order to improve the percentage of informed vehicles is not suitable when dealing with safety applications.

During the design of broadcast message dissemination schemes, it should be noted that they are remarkably influenced by the radio signal attenuation caused by the separation of sending vehicles and receivers, especially in areas with low vehicle densities, by the effect of obstacles like buildings that frequently block signal transmission in urban areas, and by the instant density of vehicles.

In fact, the map topology is very important for VANETs since it directly influences the mean distance among communicating vehicles and the presence of obstacles. Additionally, the density of vehicles clearly affects the alert message dissemination protocols since lower densities can lead to packet losses due to poor communications, and higher densities usually lead to broadcast storms [5], that is, the effect of reducing the efficiency of packet delivery due to massive contention, message repetitions, and packet collisions.

Existing dissemination schemes can be classified into one-hop or multihop schemes depending on whether or not warning message forwarding is allowed. Figure 4 presents a taxonomy of the broadcast schemes analyzed. As shown, most of the proposals rely on multihop techniques. In this group we can also consider two different categories: (i) the restrictive schemes and (ii) the promiscuous schemes. Regarding restrictive schemes, since multihop schemes usually present broadcast storm problems, several authors have proposed dissemination schemes specially designed to overcome this issue. As for the promiscuous schemes, due to the lack of infrastructure and the high mobility of the vehicles, VANETs can also present disconnected vehicles. Schemes that fall into this category try to solve this problem by using techniques such as Store and Forward to ensure that information is correctly disseminated. In the next subsections, we present all these approaches in detail.

4.1. One-Hop Dissemination Schemes. One-hop messages are those periodically exchanged by neighbor vehicles and that are not forwarded to other vehicles.

The IEEE 1609.4 standard based on the 802.11p amendment manages multichannel operations at $5.9 \mathrm{GHz}$ band. More specifically, it divides the available band into seven channels of $10 \mathrm{MHz}$ bandwidth. In particular, there are a Control Channel, two channels for special uses at the end of the frequency band, and four Service Channels ready for safety and nonsafety applications [34]. One-hop safety messages using this standard are generated periodically at a typical rate of $10 \mathrm{~Hz}$ in VANETs to provide updated information about traffic conditions.

Some works regarding single-hop safety broadcasting in vehicular networks can be found in the literature. Next, some of the most relevant ones are presented. 


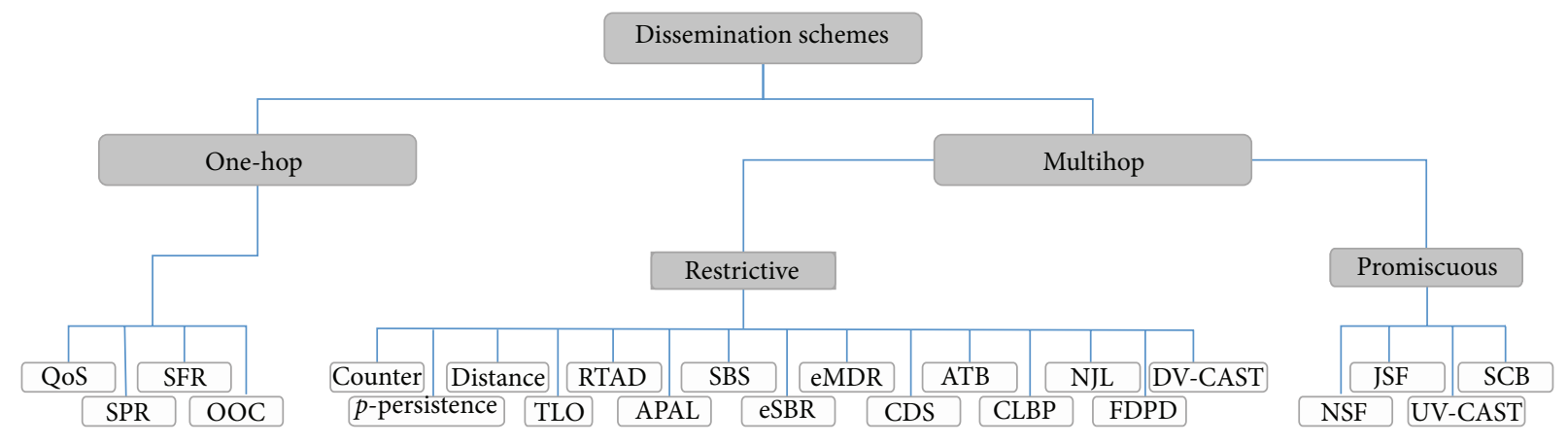

Figure 4: Taxonomy of different schemes analyzed.

(i) Xu et al. [35] proposed a model defining Quality-ofService (QoS) for safety messages using the $802.11 \mathrm{p}$ standard. This scheme favors a high reception probability for warning messages in terms of vehicles within direct communication range. The delivery time of a single message is used as a time slot, and several slots are used to define a time frame. However, in order to increase the likelihood of successful reception, messages need to be rebroadcasted multiple times within their lifetime since their range is limited to one-hop neighbors. A similar procedure is used in [36], where vehicles send short, brief messages requiring rapid repetition to achieve high reliability and low delay.

(ii) Torrent-Moreno et al. [37] studied how to manage power control in VANETs in scenarios with high vehicular density, and when broadcasting singlehop safety messages, in particular, they limited the channel load by means of a fairness criterion. However, only simple straight road scenarios are used to evaluate the proposed solution, achieving optimistic performance results.

(iii) Farnoud and Valaee [38] investigated different patterns for one-hop safety message retransmission: Synchronous Fixed Retransmission, Synchronous $p$ Persistent Retransmission, and Optical Orthogonal Codes. In particular, they showed that the latter is able to increase success probability and reduce delay. The simulation results were obtained in a 3-lane straight road, thus not being completely relevant for urban scenarios where wireless signals tend to be blocked by obstacles (e.g., buildings).

(iv) Hassanabadi and Valaee [39] presented a modification of the application layer specially designed to support safety applications using single-hop safety messages. However, it is necessary to rebroadcast the same messages several times to improve the overall reliability, making it necessary to include additional mechanisms to address well-known problems such as synchronized collisions, channel loss, and network congestion.

(v) Park and Kim [40] addressed collision control for safety applications in VANETs requiring message rates above $10 \mathrm{~Hz}$. A new application-level control algorithm was designed to modify the transmission time of one-hop messages to increase the message reception probability. Since frequency adaptations are not allowed due to the application requirements, the transmission phase was modified to increase the performance of the system.

In general, dissemination schemes based on single-hop safety messages provide local information, hence requiring additional aggregation algorithms to be feasible in safety applications covering a wide area, which limits their functionality in such scenarios. These operations increase the computational overhead of the applications, which may delay the detection and notification of dangerous situations, thus making them unsuitable in many scenarios. In addition, most of the schemes available in the literature are only evaluated in very simple scenarios without any obstacles, which is prone to generate overly optimistic results.

Considering the issues mentioned above, we now focus on multihop broadcast schemes where vehicles behave in two different modes: warning mode vehicles, which are those directly detecting dangerous situations and acting as sources of safety messages, and normal mode vehicles, which act as message relays, allowing widespread dissemination of an event in the area of interest.

4.2. Multihop Dissemination Schemes. In vehicular networks, when a vehicle detects a potentially dangerous situation, it immediately sends a warning message to its neighbors. This message will be rebroadcasted by receiving vehicles (in a multihop fashion) to notify nearby vehicles of this situation, thereby avoiding additional risks.

In this section, we present some of the most suitable multihop broadcast schemes proposed to deliver alert messages (e.g., in case of an accident), to advertise critical situations on the road, or those situations having similar requirements and that can equally benefit from this type of solution.

(i) The counter-based scheme proposed by Tseng et al. [5] was initially proposed for MANETs. More specifically, this scheme monitors the number of receptions of a broadcast packet by means of a counter $c$ and a threshold $C$. If $c \geq C$ for a received message, rebroadcast is not allowed. 
(ii) In the distance-based scheme [5], the rebroadcast of a message is determined by the distance $d$ between sending and receiving vehicles. In particular, it is not recommended to rebroadcast it when vehicles are closer, since the additional coverage (AC) obtained by doing so is low and the maximum benefit of forwarding is achieved when the additional coverage is maximized [5].

(iii) The slotted p-persistence and the weighted ppersistence schemes proposed by Wisitpongphan et al. [41] are broadcast storm mitigation techniques based on probabilities, where vehicles with a higher priority are allowed to use the channel in the least possible time. These techniques are among the few rebroadcast techniques conceived specifically for broadcast storm alleviation in VANETS, although their particular design makes them mostly suitable for highway scenarios since performance problems emerge in urban scenarios.

(iv) The Last One (TLO) is a scheme proposed by Suriyapaibonwattana and Pomavalai [42] where whenever a vehicle sends a warning message, there is a search process to locate the farthest reachable vehicle, which will be the only one granted to forward the packet. The distances between the sender and the rest of receiving vehicles are computed by means of positioning information gathered by GPS devices. This method is simple and enhances performance when compared to simple rebroadcasting, but since it does not account for urban obstacles like buildings in wireless communications, it is only effective in highway environments. In addition, it is unclear how vehicles are able to estimate the position of neighbor nodes when this information is needed.

(v) The Adaptive Probability Alert Protocol (APAL) is an extension to the TLO scheme including adaptive wait-windows and introducing different transmission probabilities [43]. This scheme outperforms TLO, but it still presents the same limitations regarding the situations where it is applicable, being only assessed in simple highways.

(vi) The stochastic broadcast scheme (SBS) was presented by Slavik and Mahgoub [44] with the goal of obtaining anonymity and scalability. In particular, nodes use a retransmission probability function to forward messages. The behavior of this scheme is affected by the vehicle density, and so this probability needs to be tuned for each specific scenario. Additionally, SBS was only tested in obstacle-free scenarios, and the influence of buildings on radio signal propagation has not been studied so far.

(vii) The enhanced Street Broadcast Reduction (eSBR) [45] uses the information obtained from the maps and the GPS to enhance alert message delivery in VANETs. One of the following conditions must be fulfilled for a vehicle to rebroadcast: (i) it must be located far away from the sender $\left(>d_{\min }\right)$, or (ii) the receiving vehicle is located in a different street, thus accessing to other areas of the map. eSBR uses the roadmap data to overcome blind areas since buildings usually block the wireless signal, preventing the communication among vehicles.

(viii) Fogue et al. presented the enhanced Message Dissemination for Roadmaps (eMDR) [46], which is an extension to eSBR. The eMDR scheme attempts to reduce even more the amount of messages produced by avoiding to rebroadcast the same warning message multiple times. Information about the junctions present in the roadmap is used, so that only one of the vehicles located in each junction is allowed to forward the warning message (specifically, the closest node to the center of the intersection in the map). Authors show that this mechanism is able to diminish the number of rebroadcasts required without reducing the rate of vehicles receiving warning messages.

(ix) The Connected Dominating Set (CDS) proposed by Ros et al. [47] employs periodic beacon messages to compute information about local positions in order to enhance the dissemination process. In particular, these beacons are used to determine whether the vehicles belong to a CDS in order to benefit from shorter retransmission waiting periods. Broadcast messages identifiers are included into the beacons as piggybacked acknowledgments. Therefore, after the expiration of the waiting timeout, the messages are retransmitted by vehicles in case that one of their neighbors did not acknowledge their correct reception.

(x) Sommer et al. presented the Adaptive Traffic Beacon (ATB) [48], a message dissemination protocol which is completely distributed and employs two key metrics to adapt beaconing: channel quality and message utility. Results showed that, compared to floodingbased approaches, adaptive beaconing provides better dissemination, although at a slower rate. The goals of this scheme are twofold: sending beacons as often as possible so as to exchange information contained in knowledge bases and achieving a congestion-free wireless channel.

(xi) Bi et al. proposed the Cross Layer Broadcast Protocol (CLBP) [49], a dissemination scheme that selects appropriate forwarding vehicles considering (i) the channel conditions, (ii) the geographic positions, and (iii) speed of cars. Reliable transmissions in CLBP are achieved by sending Broadcast Request To Send and Broadcast Clear To Send messages. The CLBP has the goal of reducing the transmission delay, but it is only designed to work in single-direction and highway scenarios. In addition, it has not been tested in urban environments.

(xii) The Nearest Junction Located (NJL) is a warning message dissemination scheme proposed by Sanguesa et al. [50] that was designed for VANETs communications in urban environments. In particular, the 
only vehicles allowed to forward warning messages are those located closer to the geographic coordinates of any junction in the map, obtaining this information from positioning devices. The NJL scheme shares this working mode with the eMDR algorithm, although only the topology and location information of the receiving vehicles are used. As expected, this scheme does not provide optimal performance in sparse scenarios. In particular, the best results are obtained in environments presenting a high density of vehicles, where NJL drastically reduces broadcasts while keeping similar results comparable to the eMDR and eSBR schemes.

(xiii) The Junction Store and Forward (JSF) proposed by Sanguesa et al. [51] was specially designed to make use of the topology characteristics and the effect of obstacles in wireless communications, since it considers that vehicles should wait to be near the crossings to rebroadcast alert messages. Unlike other existing proposals that immediately allow vehicles to forward received warning messages, according to the JSF protocol vehicles can store warning messages until a better communicating situation arises. This scheme requires each vehicle to maintain a neighbor list, which is updated taking advantage of the beacons exchanged by the cars, as well as the information provided by the GPS to decide if a vehicle is near an intersection.

(xiv) In an attempt to maximize the performance of the Store and Forward approach in sparse urban environments, the Neighbor Store and Forward (NSF) scheme [52] is a solution that, similar to JSF, requires a neighbor list to be updated by means of onehop beacons spread among vehicles; however, instead of using information about the roadmap, NSF only relies on neighbor information. Similar to JSF, after receiving a warning message, each vehicle determines whether there are additional neighbor vehicles before rebroadcasting the message. After the message is stored, the vehicle waits until it finds a new neighbor to rebroadcast the message, that is, until it receives a beacon from another car which is not contained in the neighbor list. The neighbor list is then updated, and stored messages are forwarded to inform the new neighbor about the dangerous situation. The approach followed by this scheme is different from the one used to develop the JSF scheme. While JSF focuses on informing new areas of the topology by means of additional retransmissions at street junctions, NSF is designed to inform new vehicles as soon as they arrive at the affected area.

(xv) The Store-Carry-Broadcast (SCB) scheme is proposed by Sou and Lee [53], which improves the dissemination of messages accounting for a specific road segment instead of individual vehicles. According to this dissemination scheme, warning messages are stored, carried, and broadcasted by vehicles traveling in the reverse lane to assist message dissemination.
Comparing its performance with the well-known store-carry-forward scheme, results show that SCB is able to reduce bandwidth consumption by limiting the number of broadcasts performed.

(xvi) Tonguz et al. [54] presented the Distributed Vehicular Broadcast (DV-CAST) protocol. Specifically, DVCAST is based on information about local topology. DV-CAST alleviates the broadcast storm and the disconnected network problems simultaneously, without significantly increasing the additional overhead. In particular, the DV-CAST protocol accounts for neighbors to decide whether messages should be rebroadcasted by adapting the dissemination process based on the density of neighbor vehicles, their position, and their direction.

(xvii) Viriyasitavat et al. [55] proposed the Urban Vehicular broadcast (UV-CAST) protocol to reduce broadcast storms while solving communication problems in urban scenarios. The UV-CAST algorithm selects different mechanisms for message dissemination in VANETs, differentiating between well-connected and disconnected network scenarios. Vehicles in wellconnected regimes rebroadcast incoming messages after a waiting time if no redundant messages are received. Vehicles under disconnected regimes must decide if they are suitable for storing the message and forward it whenever they meet new neighbors. Only the vehicles that are expected to find new neighbors in a short time period will be allowed to store, carry, and forward messages.

(xviii) Sormani et al. [56] proposed a function designed for message propagation. More specifically, it considers data about target zones for the messages, as well as selected routes. Then they evaluated the effectiveness of this function using different routing protocols. In addition, they proposed the Function-Driven Probabilistic Diffusion (FDPD), a probabilistic message dissemination protocol which makes use of a propagation function calculated using the separation between communicating vehicles. The given function tries to determine which vehicles are the most suitable for forwarding messages to alleviate broadcast storms.

(xix) Real-time Adaptive Dissemination (RTAD) [57] is an algorithm that selects the optimal broadcast scheme for each VANET scenario based on both the percentage of informed vehicles, which is a key parameter for the proper dissemination of warning messages, and the amount of messages received by each car in the scenario, which is used as a metric to estimate the channel contention in the warning message dissemination process.

4.3. Classification of Multihop Dissemination Schemes. In vehicular networks, message dissemination is critical to quickly inform vehicles about problems that may affect them. However, massive dissemination of messages is prone to 


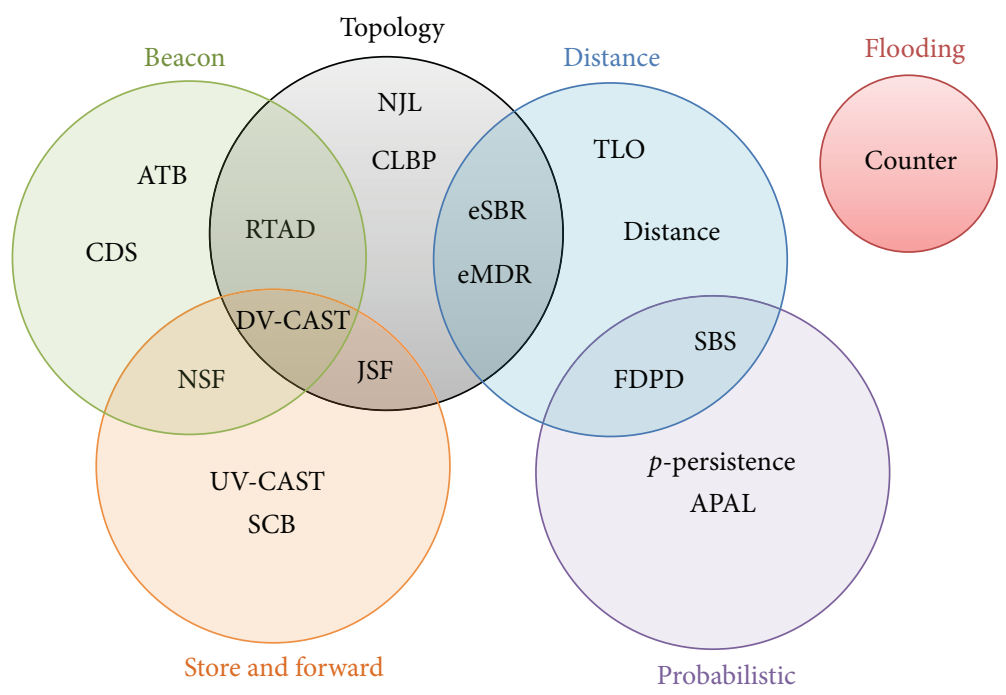

FIGURE 5: Venn diagram classifying the multihop broadcast dissemination schemes studied according to the dissemination policy adopted.

cause broadcast storm problems if no mechanisms are introduced to prevent it. Most dissemination schemes mitigate broadcast storms by refraining certain nodes (i.e., vehicles) from rebroadcasting using different parameters, thereby reducing contention in the channel, as well as message redundancy and collisions.

Figure 5 presents the proposed classification of the dissemination schemes presented above. In particular, we classified them according to their different characteristics and to techniques they use to determine whether a vehicle is allowed to rebroadcast a message (i.e., beacon-based, topologybased, distance-based, flooding-based, probabilistic-based, and Store and Forward techniques). Next, we present them in detail.

(i) Flooding. It is a very simple policy that works by making nodes directly rebroadcast all the messages received. We consider that the counter-based dissemination scheme is part of this group (i.e., a limited flooding) since this approach monitors the number of receptions of a broadcast packet by means of a counter $c$ and a threshold $C$. If $c \geq C$ for a received message, rebroadcast is not allowed for that message.

(ii) Beacon. In vehicular networks, similar to other wireless networks, beacons are periodic messages sent by vehicles with information regarding their positions, speed, and so forth. When using safety applications, beacons have lower priority compared to alert messages. Additionally, they are not forwarded by neighbors. However, the information contained by these messages could be used by vehicles to improve the knowledge about their surrounding area, taking decisions accordingly. In this category we found several proposals such as ATB, CDS, RTAD, DVCAST, and NSF. All of them use the received beacons to determine whether to rebroadcast a message. (iii) Topology. As expected, topology constrains cars' movements, so it greatly affects simulations of vehicle mobility. Moreover, it also influences the mean separation between communicating vehicles and the presence of barriers (i.e., buildings). Considering that the impact of urban obstacles like buildings on the radio signal propagation is of utmost importance in realistic urban scenarios, the information regarding the road topology can be used to maximize the propagation performance (e.g., vehicles placed at suitable locations are usually the only ones allowed to forward messages). Several broadcast dissemination schemes, such as NJL, CLBP, eSBR, eMDR, RTAD, DV-CAST, and JSF, use the topology-related information to improve the dissemination process.

(iv) Distance. According to this technique, the rebroadcast of a message is determined depending on the separation $d$ between sender and receiver vehicles. In particular, it is not recommended to rebroadcast a message when the distance separating these vehicles is reduced since the expected additional coverage (AC) obtained by doing so is low [5]. The additional coverage will increase with $d$, improving the usefulness of messages forwarded under these circumstances. Several proposed schemes, such as TLO, distancebased, SBS, eSBR, eMDR, and FDPD fall into this category.

(v) Store and Forward. In this category, once a new alert message is received, the car stores it and then waits to rebroadcast the message until a given criterion, which determines when the package should be sent, is fulfilled. According to this technique, a vehicle usually waits to rebroadcast the message until a new neighbor is found, trying to maximize the performance, especially in sparse environments. Several proposed schemes, such as UV-CAST, SCB, DV-CAST, JSF, and NSF, belong to this category. 
TABLE 1: Parameters selected to assess the different broadcast schemes.

\begin{tabular}{|c|c|c|c|c|c|c|}
\hline Scheme & Topology & RPM & Max. Tx range & Standard & Mobility model & Simulator \\
\hline Counter [5] & $0.25-25 \mathrm{~km}^{2}$ field & Free space & $500 \mathrm{~m}$ & 801.11 & RWP & Custom $\mathrm{C}++$ simulator \\
\hline Distance [5] & $0.25-25 \mathrm{~km}^{2}$ field & Free space & $500 \mathrm{~m}$ & 801.11 & RWP & Custom $\mathrm{C}++$ simulator \\
\hline eMDR [46] & $4 \mathrm{~km}^{2}$ urban & RAV & $400 \mathrm{~m}$ & $802.11 \mathrm{p}$ & Krauss & ns- 2 \\
\hline p-persistence [41] & Single and multilane & Free space & $1000 \mathrm{~m}$ & $802.11 \mathrm{a}$ & - & OPNET \\
\hline TLO [42] & Four-lane street & - & $300 \mathrm{~m}$ & 802.11 & Uniform speed & GrooveNET \\
\hline APAL [43] & Four-lane street & - & $200 \mathrm{~m}$ & $802.11 \mathrm{~b}$ & Uniform speed & GrooveNET \\
\hline SBS [44] & $1 \mathrm{~km}^{2}$ field & - & $10 \mathrm{~m}$ & - & - & Custom Java simulator \\
\hline CLBP [49] & Two-line highway & TRG & $250 \mathrm{~m}$ & 802.11e & Constant speed & ns- 2 \\
\hline NJL [50] & $4 \mathrm{~km}^{2}$ urban & RAV & $400 \mathrm{~m}$ & 802.11p & Krauss & ns-2 \\
\hline RTAD [57] & $4 \mathrm{~km}^{2}$ urban & RAV & $400 \mathrm{~m}$ & 802.11p & Krauss & ns-2 \\
\hline FDPD [56] & $4 \mathrm{~km}^{2}$ Manhattan & TRG & $200 \mathrm{~m}$ & 802.11 & Manhattan & J-Sim \\
\hline UV-CAST [55] & $1 \mathrm{~km}^{2}$ urban & LOS & $140-250 \mathrm{~m}$ & $802.11 p$ & CA-based & ns-2 \\
\hline DV-CAST [54] & Circular highway & Ricean & - & 802.11a & Uniform speed & ns-2 \\
\hline JSF [52] & $4 \mathrm{~km}^{2}$ urban & RAV & $400 \mathrm{~m}$ & $802.11 \mathrm{p}$ & Krauss & ns-2 \\
\hline
\end{tabular}

(vi) Probabilistic. The schemes included in this category require using probabilistic distributions to determine the probability of broadcasting a given message, depending on the conditions of the transmitting vehicle. Most of the schemes that fall in this category make use of the Gaussian or the uniform distribution to associate a probability to each message or vehicle. In this category, we found several proposed schemes such as FDPD, SBS, APAL, and $p$-persistence approaches.

As shown, most of the existing broadcast schemes only account for a specific characteristic or only consider a single technique (e.g., ATB, CDS, UV-CAST, SCB, or distancebased). However, other approaches such as DV-CAST, RTAD, JSF, eSBR, eMDR, and FDPD combine two different elements to improve dissemination performance (e.g., beacons and topology, topology and Store and Forward techniques, and distance and probabilistic functions). In general, this way to proceed seems to be better since the more the information is used to make a rebroadcast decision, the higher the probability of making the optimal decision is.

\section{Parameters Applied to Assess the Performance of the Schemes Studied}

One of the challenges that researchers should address when assessing their new proposals is to compare them against other similar approaches. However, it is difficult to determine which approaches present better performance, especially when noticing that existing approaches are typically validated under very different environments and that sometimes the simulation parameters are not very realistic, making the conclusions obtained inaccurate and nonrepresentative. In this section, we discuss the different configurations used by researchers when evaluating their proposals.

Table 1 shows the parameters used by authors when assessing the performance of their proposed broadcast dissemination schemes (i.e., topology, radio propagation model, maximum transmission range, etc.). We consider that they are important parameters that may affect the results obtained. However, we observed that the chosen parameters greatly vary from one work to another and also the simulation environment used, making it difficult to determine which proposal is the optimal one in each specific scenario. Next, we present the different parameters in detail.

5.1. Topology. Topology is an important factor since it directly affects mobility and communication capabilities. In particular, the topology constrains vehicles' movements and it also affects wireless signal propagation (especially in urban environments and at high radio frequencies). In VANET research, the topology of the simulated map can be manually defined by researchers, arbitrarily generated by simulators, or directly gathered from databases, such as TIGER [58] or OpenStreetMap [59].

As expected, using complex roadmaps requires more hardware resources and simulation time, although results acquired will be very accurate (i.e., closer to reality). However, we observe that simulated maps usually involve simple highways (without junctions) or a Manhattan-style map (where streets are orthogonally arranged). Although these layouts can be very easily simulated, from our perspective, more realistic scenarios should be adopted whenever possible to guarantee that the results obtained resemble those obtained in real environments.

5.2. Radio Propagation Model. As for the radio propagation model (RPM), we find that the majority of the broadcast dissemination proposals did not use RPMs offering enough accuracy for vehicular environments [60]. More specifically, the effect of existing obstacles in signal propagation (e.g., buildings) is usually omitted, which is clearly unrealistic, and surely will affect the accuracy of the results obtained.

According to data presented in Table 1, we observe that different RPMs and maximum transmission ranges have been used when assessing broadcast dissemination approaches. 
(i) Free Space Model [61]. This radio propagation model considers that the propagation conditions are ideal by assuming that there are no obstacles, and only one path between the sender and the receiver exists.

The free space radio propagation model essentially considers that all the nodes within the maximum communication range will receive all transmitted messages. However, the presence of obstacles such as buildings cannot be neglected in vehicular networks, especially in urban environments.

(ii) Two-Ray Ground (TRG) Model [62]. Unlike the Free Space model, the TRG reflection model accounts for both the direct and the ground reflection paths. This model provides a more accurate prediction than the Free Space model when considering longer distances. However, similar to the Free Space model, it overlooks several issues such as wireless signal attenuation due to obstacles.

(iii) Line-of-Sight (LOS) Dependent [63]. This propagation model is based on the TRG. In particular, this model uses the TRG considering a maximum communication range of 250 meters when sender and receiver are in LOS, whereas it only considers a maximum transmission range of $140 \mathrm{~m}$ when an obstacle prevents the LOS.

(iv) Ricean Fading. It is a probabilistic radio propagation model which accounts for deviations provoked by an imperfect radio signal. In particular, this model considers multipath interference commonly caused by the stronger signal (i.e., the line-of-sight).

(v) Real Attenuation and Visibility (RAV) [64]. This approach allows increasing the accuracy of vehicular simulations, especially in real urban roadmaps. In particular, it considers that the wireless signal will mostly be affected by the distance between communicating vehicles and the presence of obstacles between them.

5.3. Communication Standards. Regarding communication standards, the majority of proposals, fortunately, have been validated under the $802.11 \mathrm{p}$ standard, since it is expected to be globally adopted. Therefore, new approaches related to vehicular networks should account for 802.11 p specifications. Notice that this standard provides a detailed description to guarantee communication among vehicles by accounting for the special characteristics of the vehicular environment.

5.4. Mobility Model. Another determinant factor in terms of performance and representativeness of the results is the mobility model [65], which should provide a realistic and accurate mobility description at different levels (i.e., macroscopic and microscopic) [13]. In particular, mobility models attempt to closely depict the mobility patterns of drivers. Therefore, researchers should carefully select a realistic mobility model in their vehicular simulations, especially when evaluating the vehicular ad hoc communication performance [66].
More specifically, to perform realistic vehicular simulations and thus better assessing new proposals, it is important to rely on a detailed microscopic traffic simulator. Additionally, it has been demonstrated that mobility models can affect the results obtained in a decisive manner [67].

According to data presented in Table 1, we observe that the following mobility models have been used:

(i) The Random Waypoint (RWP) Model, commonly used in mobile ad hoc networks (MANETs) [68]. However, the need for a road model since, in vehicular networks, mobility is constrained by the streets is widely assumed. Additionally, vehicles cannot move independently from others; in particular, they move according to well-established traffic rules. Therefore, MANET-specific mobility models not are suitable for VANETs.

(ii) Constant Speed and Uniform Speed (USM) Models. A very simple mobility model is the Constant Speed model, which considers that each vehicle moves at a constant speed $v$. According to the USM model, vehicles are allowed to increase their speed and even overtake other vehicles. Although this kind of models can be useful in highway scenarios, they could provide unrealistic results in urban scenarios.

(iii) The Manhattan Model [69]. It is a model which only accounts for grid road topologies. Additionally, it determines the vehicles' movements according to a probabilistic function. In particular, at each intersection, vehicles should decide to keep going in the same direction or to turn left or right according to different associated probabilities. Unlike other mobility models, it does not resemble typical drivers' behavior.

(iv) The Krauss Mobility Model [70]. It accounts for collision avoidance by adapting the speed of vehicles to the speed of their predecessors, something desirable when simulating realistic traffic performance.

(v) The CA-Based Mobility Model. The cellular automata approach used to assess UV-CAST was initially presented in [71]. Despite its ease of implementation and simplicity, this model considers an accurate intersection control mechanism while providing realistic vehicle turning rules. Although the CA model can accurately reproduce the traffic flow, especially in urban environments, it still allows real-time microscopic simulations of very large networks.

5.5. Simulator Used. Exhaustive VANET simulations should involve the testing of different and heterogeneous scenarios. Compared to MANETs, the simulation of VANETs must consider the particular characteristics present in vehicular environments. The growing popularity of vehicular networks has inspired researchers to implement more realistic and precise simulation frameworks. In general, they all show good simulation capabilities, but their scalability is poor and some of them are not user-friendly.

According to data presented in Table 1, we observe that the most widely used simulator is, by far, the ns- 2 


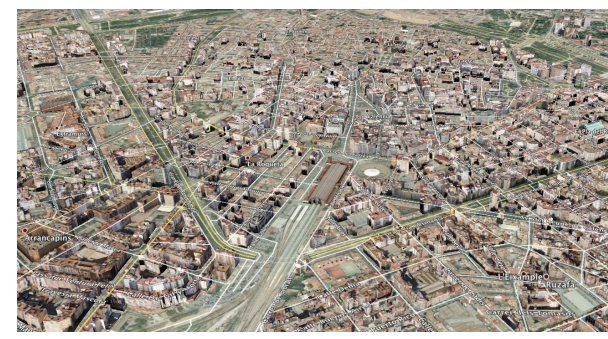

(a)

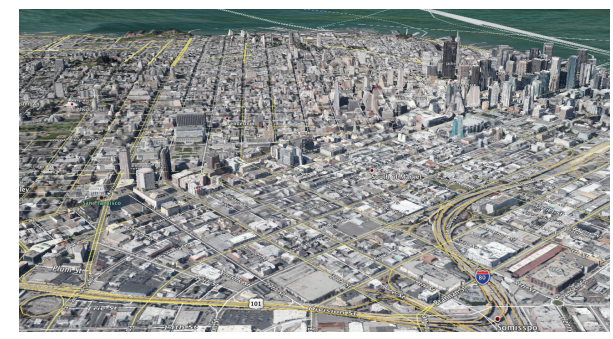

(b)

Figure 6: Maps of (a) Valencia and (b) San Francisco used in our simulations.

simulator [72], although other well-known simulators, such as OPNET [73] and GrooveNet [74], also receive much attention. The use of custom or ad hoc simulators is not a good option since results obtained may be biased, and, moreover, simulations should be easily reproduced by the research community.

Overall, we observe that some of the broadcast dissemination schemes proposed have been validated under different network simulators. Surprisingly, some of them were not specifically designed to address VANET requirements. Additionally, some of the simulation environments used did not support IEEE 802.11p, the presence of obstacles, complex urban roadmaps, or vehicular traffic models. Therefore, in this work we perform a comparative analysis of the different proposals using a realistic VANET simulation framework for the sake of accuracy and fairness.

\section{Performance Analysis}

To analyze and test the different broadcast schemes under the same conditions, we used the ns- 2 simulator, including the IEEE 802.11p standard (all these modifications can be downloaded at http://www.grc.upv.es/software/) with four channel access priorities, and the maximum broadcasting rate was set to $6 \mathrm{Mbit} / \mathrm{s}$.

Additionally, the simulator includes the Real Attenuation and Visibility (RAV) approach [64] that accounts for the presence of obstacles in the wireless signal propagation, thereby increasing the accuracy of vehicular simulations, especially in urban environments. Regarding mobility, vehicles' movements were generated by using the CityMob for Roadmaps (C4R) [75]. More specifically, C4R provides microscopic traffic capabilities, such as multilane layouts, collision free movements, lane changing, and traffic lights.

Figure 6 presents the topologies simulated, which have been gathered from the inner city areas of Valencia (Spain) and San Francisco (USA). The scenarios simulated were picked to cover topologies with distinct levels of complexity. As shown in Figure 6 and according to [50], we consider that Valencia has a complex topology and that San Francisco has a simple topology.

In our simulations, vehicles use two different broadcast modes, normal and warning mode. In particular, normal vehicles send periodic beacons with noncritical data including their position and speed. These messages are not rebroadcasted by the rest of vehicles and have low priority. Warning
TABle 2: Parameters used in the simulations.

\begin{tabular}{lc}
\hline Parameter & Value \\
\hline Map & Valencia and San Francisco \\
Vehicles per $\mathrm{km}^{2}$ & {$[25$ and 100] } \\
Collided vehicles & 3 \\
Map size & $2000 \mathrm{~m} \times 2000 \mathrm{~m}$ \\
Warning message size & $256 \mathrm{~B}$ \\
Warning messages priority & $\mathrm{AC} 3$ \\
Beacon message size & $512 \mathrm{~B}$ \\
Beacon priority & $\mathrm{AC} 1$ \\
Message interval & $1 \mathrm{~second}$ \\
MAC/PHY & $802.11 \mathrm{p}$ \\
Propagation model & RAV [64] \\
Mobility model & Krauss et al. [70] \\
Bandwidth & $6 \mathrm{Mbps}$ \\
Maximum communication range & $400 \mathrm{~m}$ \\
$d_{\text {min }}$ (distance-based, eSBR, and & $200 \mathrm{~m}$ \\
eMDR approaches) &
\end{tabular}

mode vehicles periodically send their status to other vehicles by using alert messages with high priority.

All the results in this paper were obtained as the mean of 50 random executions with a confidence level of $95 \%$. Table 2 includes the simulated parameters. As shown, we have only varied the roadmap (i.e., Valencia and San Francisco) and the density of vehicles (i.e., 25 and 100 veh. $/ \mathrm{km}^{2}$ ) since, according to [76], these are the key factors that mostly affect the performance of the warning dissemination process.

In order to assess the broadcast schemes studied, we selected the following performance metrics: (a) the portion of vehicles informed, (b) the messages received per vehicle, and (c) the warning notification time. More specifically, the portion of vehicles informed is the percentage of cars that obtain warning messages sent. The messages received per vehicle account for the overhead and channel contention of each scheme. Finally, the warning notification time measures the elapsed time from a warning message sending and its delivery to another vehicle.

During a warning message broadcast process, the main objective is to inform the highest number of vehicles as quickly as possible and without compromising the channel. 


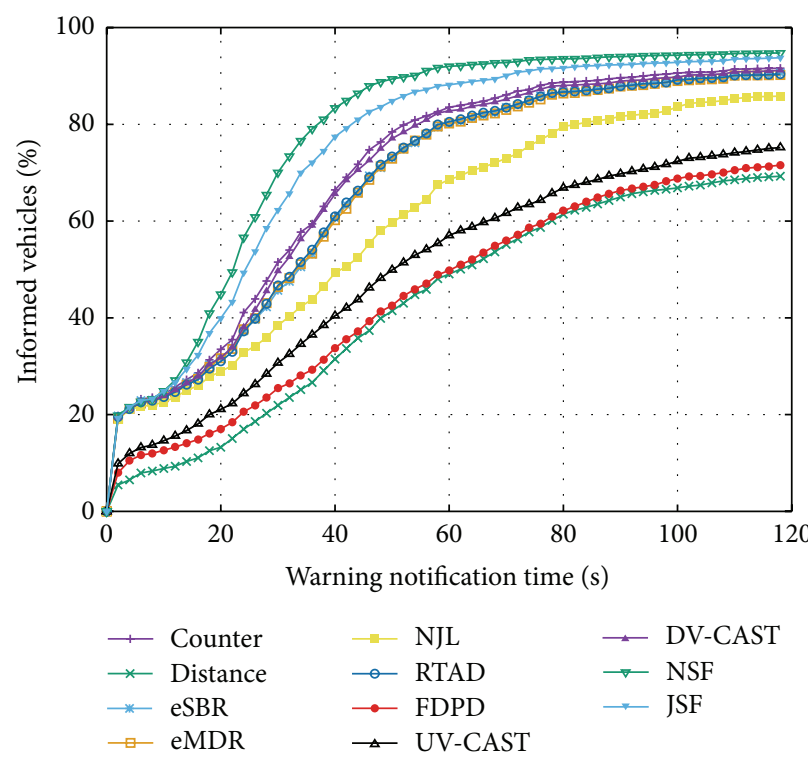

(a)

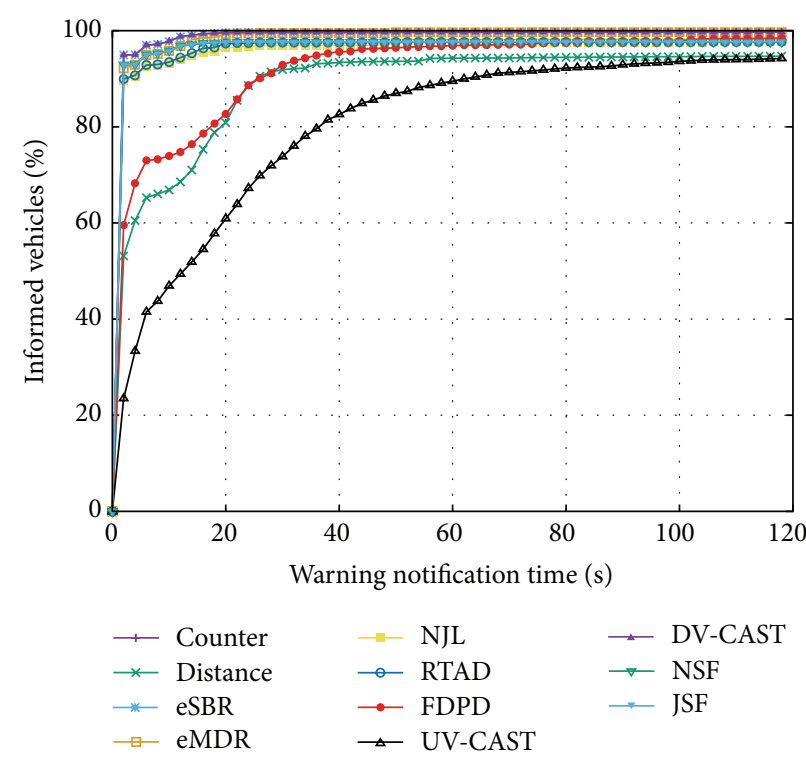

(b)

Figure 7: Percentage of vehicles informed and warning notification time in San Francisco for (a) 25 and (b) 100 vehicles $/ \mathrm{km}^{2}$.

In this section, we study the behavior of some of the most relevant broadcast dissemination schemes proposed so far. Unlike previous works, we compare all of them under the same simulation conditions, thus making it possible to determine which are the optimal ones in each situation.

Figures 7 and 8 present the dissemination behavior. In particular, we include the percentage of informed vehicles and the warning notification time for the maps of San Francisco and Valencia when simulating two different densities: 25 and 100 vehicles $/ \mathrm{km}^{2}$.

As shown, the NSF dissemination scheme achieves the highest percentage of vehicles informed in all cases, that is, under both low and high vehicle density conditions, as well as under low and high topology complexity scenarios, obtaining up to $40 \%$ additional informed vehicles compared to more restrictive dissemination approaches, such as UVCAST, FDPD, or distance-based dissemination approaches.

As for messages received per vehicle (see Figures 9 and 10), it is directly related to the performance obtained in terms of informed nodes; that is, a higher amount of messages received represents a better performance in terms of vehicles informed. However, under high densities and low complexity scenarios (see Figure 7(b)), we found that some dissemination schemes, such as RTAD, UV-CAST, eSBR, and eMDR, obtain results similar to NSF in terms of informed vehicles and warning notification time, while reducing to one-fifth the number of messages received (as shown in Figure 9(b)).

Overall, it is noticeable how the map topology and the density of vehicles are crucial factors that highly affect the performance of broadcasting. In general, the dissemination process develops faster (i.e., more vehicles are informed during the same period) when the vehicle density increases, independently from the broadcast scheme used and especially under complex roadmaps. Store and Forward methods such as NSF and JSF offer the best results in terms of informed vehicles in all the studied situations, outperforming the other schemes; however, the number of messages also increases. This increment in terms of absolute number of messages is not significant at low densities, although it could become a problem in scenarios with extremely high vehicle densities. In addition, in simple roadmaps such as San Francisco, the differences between the majority of the schemes are minimal. Hence, it would be better to use dissemination schemes which produce a lower number of messages per vehicle, such as NJL of RTAD.

\section{Lessons Learned and Guidelines for Future Research}

Taking into account all the information related to the warning message dissemination mechanisms presented along this paper (different features, vehicular simulation environments, dissemination performance, etc.), we summarized in Table 3 the main pros and cons of the different broadcast dissemination schemes studied.

As shown, most existing schemes rely on GPS information alone to select the next forwarding vehicles. This requirement is feasible since, in modern Intelligent Transportation Systems (ITS), vehicles incorporate built-in GPS systems and offline maps. Therefore, vehicles are able to acquire data related to their speed, acceleration, position, and so forth, in order to broadcast this information to their neighbors. When neighboring vehicles receive this data, they can extract useful information to detect and avoid potential risks. Moreover, both topology and location information can be used by the warning message dissemination schemes to enhance their performance. Despite the fact that GPS information should be as accurate as possible, especially when the schemes must determine exactly when a vehicle is at an intersection or even 


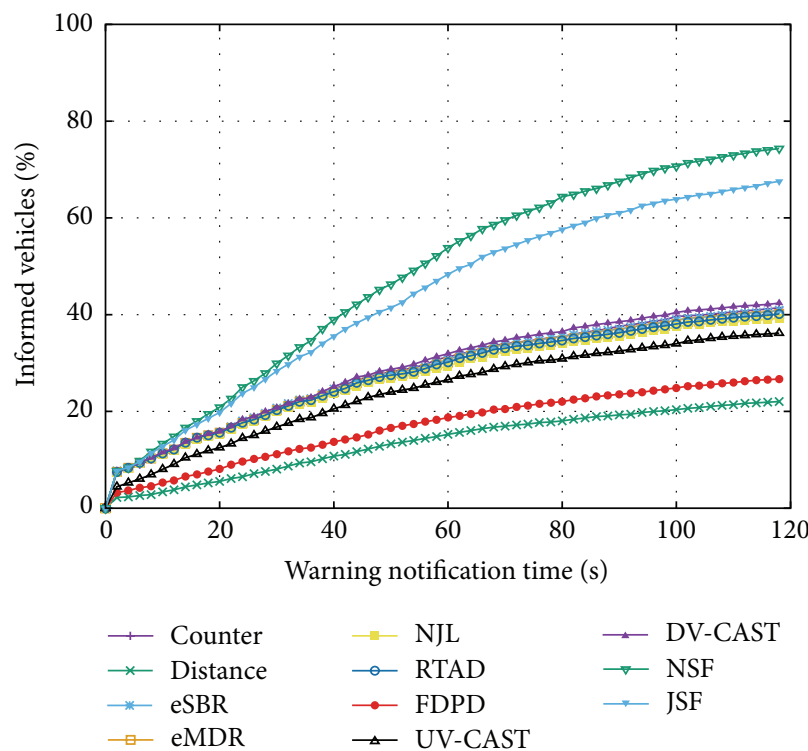

(a)

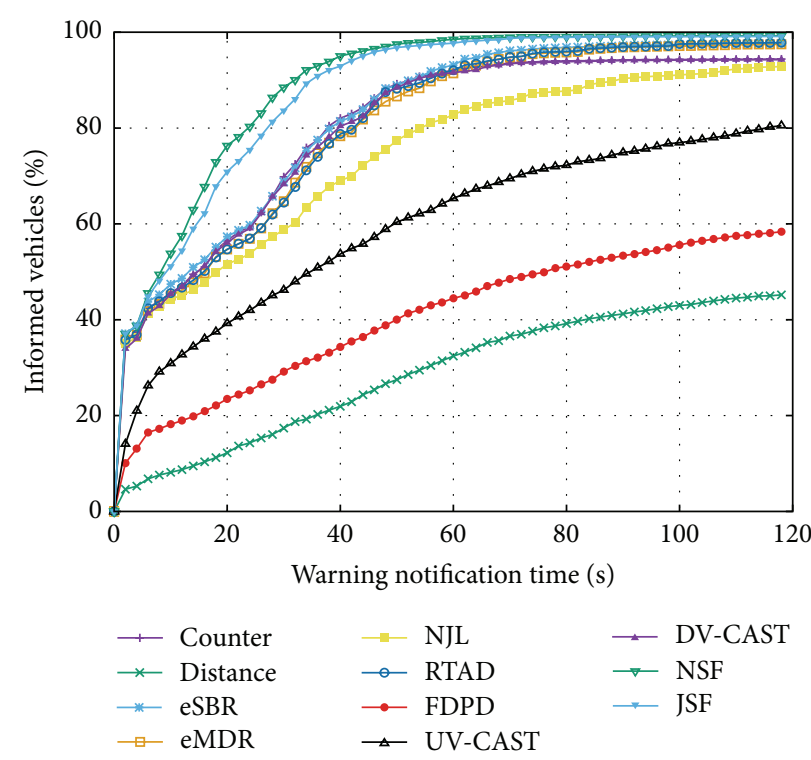

(b)

Figure 8: Percentage of vehicles informed and warning notification time in Valencia for (a) 25 and (b) 100 vehicles $/ \mathrm{km}^{2}$.

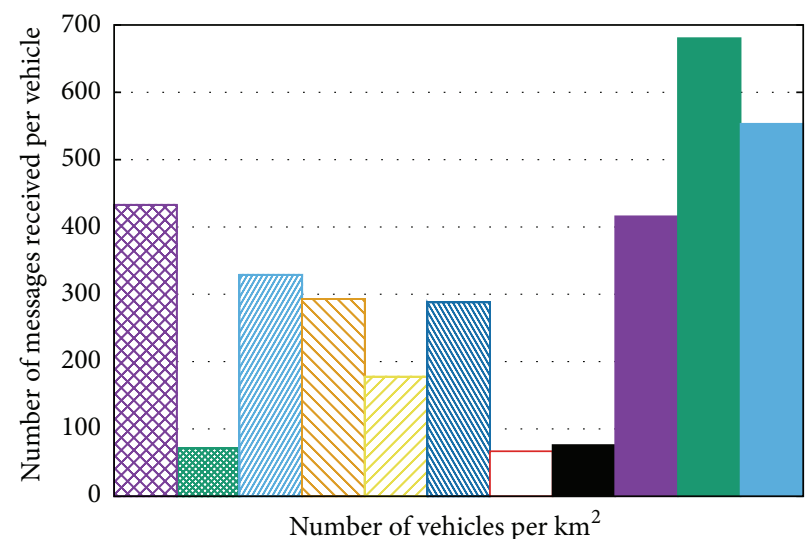

$\begin{array}{lll}\text { Counter } & \text { NJL } & \text { DV-CAST } \\ \text { Distance } & \text { RTAD } & \text { NSF } \\ \text { eSBR } & \text { FDPD } & \text { JSF } \\ \text { eMDR } & \text { UV-CAST }\end{array}$

(a)

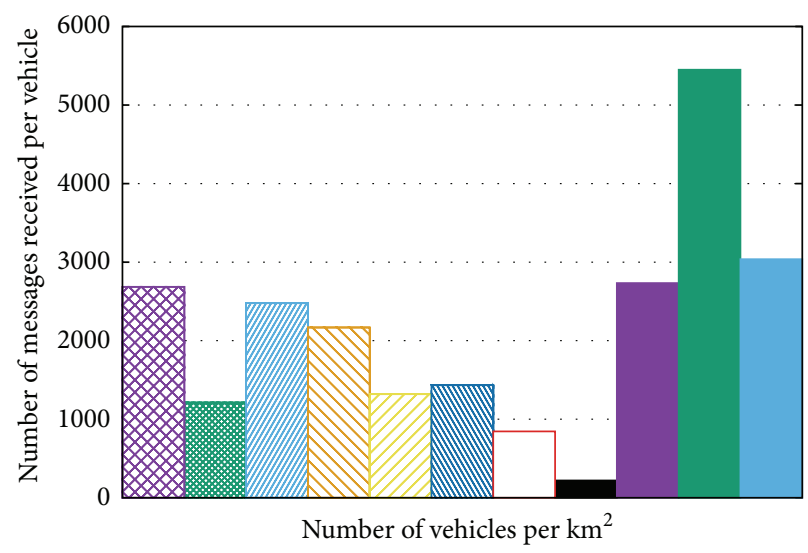

$\begin{array}{lll}\text { Counter } & \text { NJL } & \text { DV-CAST } \\ \text { Distance } & \text { RTAD } & \text { NSF } \\ \text { eSBR } & \text { FDPD } & \text { JSF } \\ \text { eMDR } & \text { UV-CAST }\end{array}$

(b)

FIGURE 9: Number of received messages per vehicle in San Francisco for (a) 25 and (b) 100 vehicles $/ \mathrm{km}^{2}$.

whether it is the one nearest to the middle of the intersection, some dissemination approaches such as [46] demonstrated that it is possible to overcome GPS inaccuracy.

In terms of efficiency, we can find approaches that are specifically designed to improve the dissemination process (e.g., counter, distance, JSF, or NSF) and other schemes which are mainly focused on reducing the broadcast storm problem, especially in vehicular urban scenarios (e.g., NJL, eSBR, or eMDR).

As for the complexity of the roadmap, some of them perform better in highway scenarios (e.g., distance, FDPD, and UV-CAST), while others are specifically designed to be used in urban environments (e.g., eSBR, eMDR, or NJL).
Finally, we can find static dissemination schemes, that is, approaches that do not change their dissemination policy (e.g., counter, distance, NJL, or FPDP) and adaptive schemes (e.g., RTAD, UV-CAST, and DV-CAST) which adapt their dissemination policy according to the current context.

We consider that future proposals related to warning message dissemination should be able to vary their dissemination policy along time since adaptive mechanisms can obtain better results than static dissemination alternatives, especially in those vehicular scenarios where conditions are frequently changing. Additionally, the use of infrastructure can improve the dissemination process. For example, Ucar et al. [77] 
TABle 3: Pros and cons of the different dissemination schemes.

\begin{tabular}{|c|c|c|}
\hline Scheme & Pros & Cons \\
\hline Counter & $\begin{array}{c}\text { Easy implementation } \\
\text { High \% of informed vehicles }\end{array}$ & $\begin{array}{l}\text { Originally proposed for MANETs } \\
\text { High number of messages used }\end{array}$ \\
\hline Distance & $\begin{array}{c}\text { Easy implementation } \\
\text { Low number of messages }\end{array}$ & Low performance in urban environments \\
\hline eSBR & $\begin{array}{l}\text { Good performance in different environments } \\
\text { Improving distance results in terms of \% of informed vehicles }\end{array}$ & GPS required \\
\hline eMDR & $\begin{array}{c}\text { Improving eSBR } \\
\text { Reducing the number of messages used }\end{array}$ & $\begin{array}{l}\text { High precision GPS required } \\
\text { Specially designed for urban environments }\end{array}$ \\
\hline NJL & $\begin{array}{l}\text { High efficiency in urban scenarios } \\
\text { Reduced number of messages used } \\
\text { Aggressive broadcast storm reduction }\end{array}$ & $\begin{array}{l}\text { High precision GPS required } \\
\text { Useless in highway scenarios }\end{array}$ \\
\hline RTAD & $\begin{array}{l}\text { Adaptive dissemination scheme } \\
\text { High efficiency in different scenarios }\end{array}$ & $\begin{array}{l}\text { Complex implementation } \\
\text { GPS required }\end{array}$ \\
\hline FDPD & $\begin{array}{l}\text { Recommended for highway scenarios } \\
\text { Direction of vehicles is considered }\end{array}$ & $\begin{array}{c}\text { GPS required } \\
\text { Low performance in urban scenarios }\end{array}$ \\
\hline UV-CAST & $\begin{array}{c}\text { Adaptive dissemination scheme } \\
\text { Connecting disconnected subnetworks } \\
\text { Reduced number of messages used }\end{array}$ & Low performance in urban scenarios \\
\hline DV-CAST & $\begin{array}{l}\text { Adaptive dissemination scheme } \\
\text { Good performance in terms of informed vehicles }\end{array}$ & $\begin{array}{c}\text { GPS required } \\
\text { Low reduction of messages } \\
\end{array}$ \\
\hline JSF & $\begin{array}{l}\text { Higher \% of informed vehicles } \\
\text { Specially indicated for simple maps }\end{array}$ & $\begin{array}{l}\text { High number of messages used } \\
\text { GPS required } \\
\text { Overhead in high density conditions }\end{array}$ \\
\hline NSF & $\begin{array}{c}\text { Highest \% of informed vehicles } \\
\text { Specially indicated for low density scenarios }\end{array}$ & $\begin{array}{l}\text { High number of messages used } \\
\text { Overhead in high density conditions }\end{array}$ \\
\hline
\end{tabular}

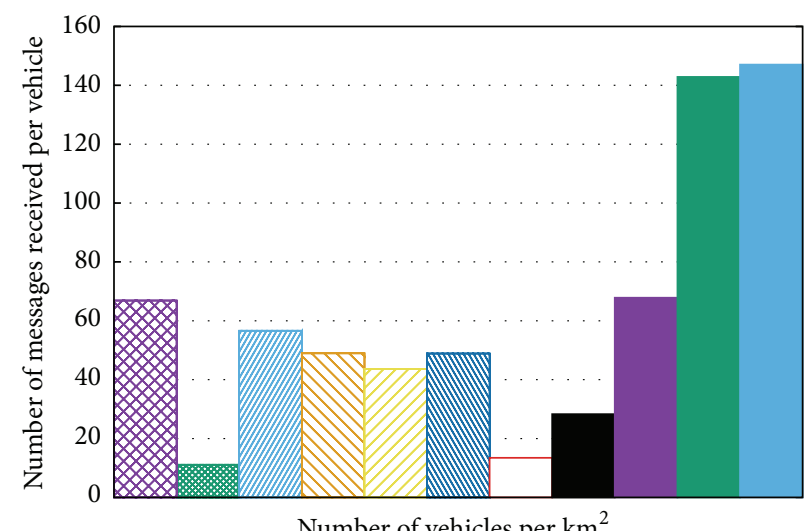

Number of vehicles per $\mathrm{km}^{2}$

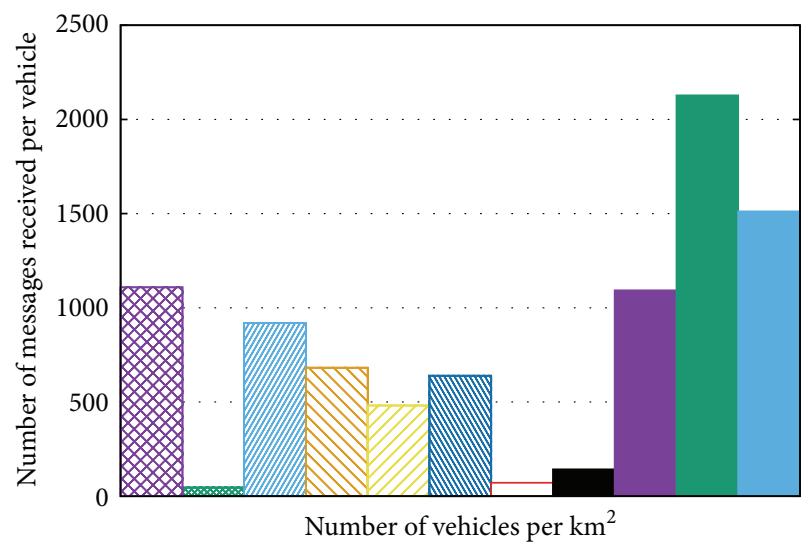

$\begin{array}{llll}\text { Counter } & \text { NJL } & \text { DV-CAST } \\ \text { Distance } & \text { RTAD } & \text { NSF } \\ \text { eSBR } & \text { FDPD } & \text { JSF } \\ \text { eMDR } & \text { UVDCAST }\end{array}$

(b) (a)

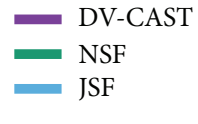

FDPD

FIGURE 10: Number of messages received per vehicle in Valencia for (a) 25 and (b) 100 vehicles $/ \mathrm{km}^{2}$.

proposed VMaSC-LTE, a hybrid architecture that combines the Long Term Evolution (LTE) and the IEEE 802.11p standards, trying to perform a higher data delivery ratio, without increasing delay, and minimizing the usage of the cellular architecture.

\section{Conclusions}

In this paper, we presented some of the most relevant broadcast dissemination schemes specially designed for VANETs, highlighting their features, and studying their performance under the same simulation conditions, thus 
offering researchers a fair comparison between different broadcast schemes.

In particular, we presented a classification of the broadcast dissemination schemes and classified them according to the different characteristics and techniques they use to determine whether a car is allowed to rebroadcast a packet. In addition, we simulated all these schemes by using a real visibility model and under realistic urban environment conditions.

According to the results obtained, we observed that Store and Forward broadcasting schemes, which account for the beacons received and the map topology, achieve a higher percentage of informed nodes, especially in sparse scenarios. However, when density increases, the high volume of messages produced is prone to saturate the channel. Additionally, we find that, as expected, adaptive dissemination schemes (such as RTAD and DV-CAST) achieve intermediate values, offering a good trade-off between the measured metrics (i.e., informed vehicles, messages received, and warning notification time) for all the vehicle densities studied.

\section{Abbreviations}

$\begin{array}{ll}\text { ACs: } & \text { Access Categories } \\ \text { APAL: } & \text { Adaptive Probability Alert Protocol } \\ \text { ATB: } & \text { Adaptive Traffic Beacon } \\ \text { BCTS: } & \text { Broadcast Clear To Send } \\ \text { BRTS: } & \text { Broadcast Request To Send } \\ \text { CCH: } & \text { Control Channel } \\ \text { CDS: } & \text { Connected Dominating Set } \\ \text { CLBP: } & \text { Cross Layer Broadcast Protocol } \\ \text { C4R: } & \text { CityMob for RoadMaps } \\ \text { DSRC: } & \text { Dedicated Short Range Communications } \\ \text { DV-CAST: } & \text { Distributed Vehicular Broadcast } \\ \text { eMDR: } & \text { enhanced Message Dissemination for } \\ & \text { RoadMaps } \\ \text { eSBR: } & \text { enhanced Street Broadcast Reduction } \\ \text { ETSI: } & \text { European Telecommunications Standards } \\ & \text { Institute } \\ \text { FDPD: } & \text { Function-Driven Probabilistic Diffusion } \\ \text { ITS: } & \text { Intelligent Transportation Systems } \\ \text { JSF: } & \text { Junction Store and Forward } \\ \text { LOS: } & \text { Light of Sight } \\ \text { MANET: } & \text { Mobile ad hock network } \\ \text { NJL: } & \text { Nearest Junction Located } \\ \text { NSF: } & \text { Neighbor Store and Forward } \\ \text { OOC: } & \text { Optical Orthogonal Codes } \\ \text { QoS: } & \text { Quality-of-Service } \\ \text { RAV: } & \text { Real Attenuation and Visibility } \\ \text { RF: } & \text { Radio Frequency } \\ \text { RTAD: } & \text { Real-Time Adaptive Dissemination } \\ \text { RPM: } & \text { Radio propagation model } \\ \text { RWP: } & \text { Random Waypoint } \\ \text { SBS: } & \text { Stochastic Broadcast Scheme } \\ \text { SCB: } & \text { Store-Carry-Broadcast } \\ \text { SCH: } & \text { Service Channel } \\ \text { SFR: } & \text { Synchronous Fixed Retransmission } \\ \text { SPR: } & \text { Synchronous p-Persistent Retransmission } \\ \text { TLO: } & \text { The Last One } \\ & \end{array}$

TRG: $\quad$ Two-Ray Ground

USM: $\quad$ Constant Speed and Uniform Speed

UV-CAST: Urban Vehicular Broadcast

VANET: Vehicular ad hock network

VN: Vehicular network

V2I: Vehicle-to-infrastructure

V2V: Vehicle-to-vehicle.

\section{Competing Interests}

The authors declare that they have no competing interests.

\section{Acknowledgments}

This work was partially supported by the Ministerio de Economía y Competitividad, Programa Estatal de Investigación, Desarrollo e Innovación Orientada a los Retos de la Sociedad, Proyectos I+D+I 2014, Spain, under Grant TEC2014-52690-R, and by the Government of Aragón and the European Social Fund (T91 Research Group).

\section{References}

[1] F. J. Martinez, C.-K. Toh, J.-C. Cano, C. T. Calafate, and P. Manzoni, "Emergency services in future intelligent transportation systems based on vehicular communication networks," IEEE Intelligent Transportation Systems Magazine, vol. 2, no. 2, pp. 620, 2010.

[2] P. Fazio, F. De Rango, and A. Lupia, "A new application for enhancing VANET services in emergency situations using the WAVE/802.11p standard," in Proceedings of the IFIP Wireless Days (WD '13), pp. 1-3, Valencia, Spain, November 2013.

[3] N. Kumar, N. Chilamkurti, and J. J. P. C. Rodrigues, "Learning automata-based opportunistic data aggregation and forwarding scheme for alert generation in vehicular ad hoc networks," Computer Communications, vol. 39, pp. 22-32, 2014.

[4] K. Shafiee, J. Lee, V. C. M. Leung, and G. Chow, "Modeling and simulation of vehicular networks," in Proceedings of the 1st ACM International Symposium on Design and Analysis of Intelligent Vehicular Networks and Applications (DIVANet '11), pp. 77-85, ACM, New York, NY, USA, November 2011.

[5] Y.-C. Tseng, S.-Y. Ni, Y.-S. Chen, and J.-P. Sheu, “The broadcast storm problem in a mobile ad hoc network," Wireless Networks, vol. 8, no. 2-3, pp. 153-167, 2002.

[6] M. Fogue, P. Garrido, F. J. Martinez, J.-C. Cano, C. T. Calafate, and P. Manzoni, "Automatic accident detection: assistance through communication technologies and vehicles," IEEE Vehicular Technology Magazine, vol. 7, no. 3, pp. 90-100, 2012.

[7] M. Fogue, P. Garrido, F. J. Martinez, J.-C. Cano, C. T. Calafate, and P. Manzoni, "A system for automatic notification and severity estimation of automotive accidents," IEEE Transactions on Mobile Computing, vol. 13, no. 5, pp. 948-963, 2014.

[8] M. Fogue, P. Garrido, F. J. Martinez, J.-C. Cano, C. T. Calafate, and P. Manzoni, "A novel approach for traffic accidents sanitary resource allocation based on multi-objective genetic algorithms," Expert Systems with Applications, vol. 40, no. 1, pp. 323336, 2013.

[9] P. Ruiz and P. Bouvry, "Survey on broadcast algorithms for mobile ad hoc networks," ACM Computing Surveys, vol. 48, no. 1, article 8, 2015. 
[10] L. Cheng, B. E. Henty, R. Cooper, D. D. Stancil, and F. Bai, "A measurement study of time-scaled 802.11a waveforms over the mobile-to-mobile vehicular channel at $5.9 \mathrm{GHz}$," IEEE Communications Magazine, vol. 46, no. 5, pp. 84-91, 2008.

[11] S. Panichpapiboon and W. Pattara-Atikom, "A review of information dissemination protocols for vehicular ad hoc networks," IEEE Communications Surveys and Tutorials, vol. 14, no. 3, pp. 784-798, 2012.

[12] X. Li and H. Li, "A survey on data dissemination in VANETs," Chinese Science Bulletin, vol. 59, no. 32, pp. 4190-4200, 2014.

[13] J. Harri, F. Filali, and C. Bonnet, "Mobility models for vehicular ad hoc networks: a survey and taxonomy," IEEE Communications Surveys \& Tutorials, vol. 11, no. 4, pp. 19-41, 2009.

[14] D. Jia, K. Lu, J. Wang, X. Zhang, and X. Shen, "A survey on platoon-based vehicular cyber-physical systems," IEEE Communications Surveys \& Tutorials, vol. 18, no. 1, pp. 263-284, 2015.

[15] S. Madi and H. Al-Qamzi, "A survey on realistic mobility models for vehicular ad hoc networks (VANETs)," in Proceedings of the 10th IEEE International Conference on Networking, Sensing and Control (ICNSC '13), pp. 333-339, April 2013.

[16] M. S. Al-Kahtani, "Survey on security attacks in vehicular ad hoc networks (VANETs)," in Proceedings of the 6th International Conference on Signal Processing and Communication Systems (ICSPCS '12), pp. 1-9, Queensland, Australia, December 2012.

[17] H. Al Falasi and E. Barka, "Revocation in VANETs: a survey," in Proceedings of the International Conference on Innovations in Information Technology (IIT '11), pp. 214-219, Abu Dhabi, United Arab Emirates, April 2011.

[18] F. Li and Y. Wang, "Routing in vehicular ad hoc networks: a survey," IEEE Vehicular Technology Magazine, vol. 2, no. 2, pp. 12-22, 2007.

[19] H. Keshavarz and R. M. Noor, "Beacon-based geographic routing protocols in vehicular ad hoc networks: a survey and taxonomy," in Proceedings of the IEEE Symposium on Wireless Technology and Applications (ISWTA '12), pp. 309-314, IEEE, Bandung, September 2012.

[20] S. Allal and S. Boudjit, "Geocast routing protocols for VANETs: survey and guidelines," in Proceedings of the 6th International Conference on Innovative Mobile and Internet Services in Ubiquitous Computing (IMIS '12), pp. 323-328, IEEE, Palermo, Italy, July 2012.

[21] A. Sebastian, M. Tang, Y. Feng, and M. Looi, "A multicast routing scheme for efficient safety message dissemination in VANET," in Proceedings of the IEEE Wireless Communications and Networking Conference (WCNC '10), pp. 1-6, Sydney, Australia, April 2010.

[22] F. Soldo, R. Lo Cigno, and M. Geria, "Cooperative synchronous broadcasting in infrastructure-to-vehicles networks," in Proceedings of the 5th Annual Conference on Wireless on Demand Network Systems and Services (WONS '08), pp. 125-132, Garmisch-Partenkirchen, Germany, January 2008.

[23] F. J. Martinez, J.-C. Cano, C. T. Calafate, P. Manzoni, and J. M. Barrios, "Assessing the feasibility of a VANET driver warning system," in Proceedings of the 4th ACM Workshop on Performance Monitoring and Measurement of Heterogeneous Wireless and Wired Networks (PM2HW2N '09), pp. 39-45, ACM, 2009.

[24] G. Y. Cahng, J.-P. Sheu, and J.-H. Wu, "Typhoon: resource sharing protocol for metropolitan vehicular ad hoc networks," in Proceedings of the IEEE Wireless Communications and Networking Conference (WCNC '10), pp. 1-5, Sydney, Australia, April 2010.
[25] X. Hu, J. Zhao, D. Zhou, and V. C. M. Leung, "A semantics-based multi-agent framework for vehicular social network development," in Proceedings of the 1st ACM International Symposium on Design and Analysis of Intelligent Vehicular Networks and Applications (DIVANet '11), pp. 87-96, ACM, New York, NY, USA, November 2011.

[26] S. Samarah, "Grid-based hierarchy structure for mining and querying vehicular ad-hoc networks," in Proceedings of the Second ACM International Symposium on Design and Analysis of Intelligent Vehicular Networks and Applications (DIVANet '12), pp. 63-68, ACM, 2012.

[27] J. Jakubiak and Y. Koucheryavy, "State of the art and research challenges for VANETs," in Proceedings of the 5th IEEE Consumer Communications and Networking Conference (CCNC '08), pp. 912-916, Las Vegas, Nev, USA, January 2008.

[28] DoT, "United States Department of Transportation," 2015, http://www.dot.gov/.

[29] Z. Movahedi, R. Langar, and G. Pujolle, "A comprehensive overview of vehicular Ad Hoc Network evaluation alternatives," in Proceedings of the 8th Asia-Pacific Symposium on Information and Telecommunication Technologies (APSITT '10), pp. 1-5, Kuching, Malaysia, June 2010.

[30] A. J. Ghandour, M. Di Felice, H. Artail, and L. Bononi, "Dissemination of safety messages in IEEE 802.11p/WAVE vehicular network: analytical study and protocol enhancements," Pervasive and Mobile Computing, vol. 11, pp. 3-18, 2014.

[31] J. Dias, J. Rodrigues, J. Isento, and J. Niu, “The impact of cooperative nodes on the performance of vehicular delaytolerant networks," Mobile Networks and Applications, vol. 18, no. 6, pp. 867-878, 2013.

[32] J. N. G. Isento, J. J. P. C. Rodrigues, J. A. F. F. Dias, M. C. G. Paula, and A. Vinel, "Vehicular delay-tolerant networks? A novel solution for vehicular communications," IEEE Intelligent Transportation Systems Magazine, vol. 5, no. 4, pp. 10-19, 2013.

[33] P. R. Pereira, A. Casaca, J. J. P. C. Rodrigues, V. N. G. J. Soares, J. Triay, and C. Cervelló-Pastor, "From delay-tolerant networks to vehicular delay-tolerant networks," IEEE Communications Surveys and Tutorials, vol. 14, no. 4, pp. 1166-1182, 2012.

[34] Q. Chen, D. Jiang, and L. Delgrossi, "IEEE 1609.4 DSRC multichannel operations and its implications on vehicle safety communications," in Proceedings of the IEEE Vehicular Networking Conference (VNC '09), pp. 1-8, Tokyo, Japan, October 2009.

[35] Q. Xu, T. Mak, J. Ko, and R. Sengupta, "Vehicle-to-vehicle safety messaging in DSRC," in Proceedings of the 1st ACM International Workshop on Vehicular Ad Hoc Networks (VANET '04), pp. 1928, ACM, New York, NY, USA, 2004.

[36] Q. Xu, T. Mak, J. Ko, and R. Sengupta, "Medium access control protocol design for vehicle-vehicle safety messages," IEEE Transactions on Vehicular Technology, vol. 56, no. 2, pp. 499518, 2007.

[37] M. Torrent-Moreno, P. Santi, and H. Hartenstein, "Fair sharing of bandwidth in VANETs," in Proceedings of the 2nd ACM International Workshop on Vehicular Ad Hoc Networks (VANET '05), pp. 49-58, New York, NY, USA, 2005.

[38] F. Farnoud and S. Valaee, "Repetition-based broadcast in vehicular ad hoc networks in Rician channel with capture," in Proceedings of the IEEE INFOCOM Workshops, pp. 1-6, Phoenix, Ariz, USA, April 2008.

[39] B. Hassanabadi and S. Valaee, "Reliable periodic safety message broadcasting in VANETs using network coding," IEEE Transactions on Wireless Communications, vol. 13, no. 3, pp. 1284-1297, 2014. 
[40] Y. Park and H. Kim, "Collision control of periodic safety messages with strict messaging frequency requirements," IEEE Transactions on Vehicular Technology, vol. 62, no. 2, pp. 843-852, 2013.

[41] N. Wisitpongphan, O. K. Tonguz, J. S. Parikh, P. Mudalige, F. Bai, and V. Sadekar, "Broadcast storm mitigation techniques in vehicular ad hoc networks," IEEE Wireless Communications, vol. 14, no. 6, pp. 84-94, 2007.

[42] K. Suriyapaibonwattana and C. Pomavalai, "An effective safety alert broadcast algorithm for VANET," in Proceedings of the International Symposium on Communications and Information Technologies (ISCIT '08), pp. 247-250, Vientiane, Laos, October 2008.

[43] K. Suriyapaiboonwattana, C. Pornavalai, and G. Chakraborty, "An adaptive alert message dissemination protocol for VANET to improve road safety," in Proceedings of the IEEE International Conference on Fuzzy Systems (FUZZ-IEEE '09), pp. 1639-1644, Jeju Island, Republic of Korea, August 2009.

[44] M. Slavik and I. Mahgoub, "Stochastic broadcast for VANET," in Proceedings of the 7th IEEE Consumer Communications and Networking Conference (CCNC '10), pp. 1-5, IEEE, Las Vegas, Nev, USA, January 2010.

[45] F. J. Martinez, M. Fogue, M. Coll, J.-C. Cano, C. Calafate, and P. Manzoni, "Evaluating the impact of a novel warning message dissemination scheme for VANETs using real city maps," in NETWORKING 2010: 9th International IFIP TC 6 Networking Conference, Chennai, India, May 11-15, 2010. Proceedings, M. Crovella, L. Feeney, D. Rubenstein, and S. Raghavan, Eds., vol. 6091 of Lecture Notes in Computer Science, pp. 265-276, Springer, Berlin, Germany, 2010.

[46] M. Fogue, P. Garrido, F. J. Martinez, J.-C. Cano, C. T. Calafate, and P. Manzoni, "Evaluating the impact of a novel message dissemination scheme for vehicular networks using real maps," Transportation Research Part C: Emerging Technologies, vol. 25, pp. 61-80, 2012.

[47] F. J. Ros, P. M. Ruiz, and I. Stojmenovic, "Reliable and efficient broadcasting in vehicular ad hoc networks," in Proceedings of the IEEE 69th Vehicular Technology Conference (VTC Spring '09), pp. 1-5, IEEE, April 2009.

[48] C. Sommer, O. K. Tonguz, and F. Dressler, "Traffic information systems: efficient message dissemination via adaptive beaconing," IEEE Communications Magazine, vol. 49, no. 5, pp. 173-179, 2011.

[49] Y. Bi, L. X. Cai, X. Shen, and H. Zhao, "A cross layer broadcast protocol for multihop emergency message dissemination in inter-vehicle communication," in Proceedings of the IEEE International Conference on Communications (ICC '10), pp. 1-5, Cape Town, South Africa, May 2010.

[50] J. A. Sanguesa, M. Fogue, P. Garrido et al., "On the selection of optimal broadcast schemes in VANETs," in Proceedings of the 16th ACM International Conference on Modeling, Analysis and Simulation of Wireless and Mobile Systems (MSWiM '13), pp. 411418, Barcelona, Spain, November 2013.

[51] J. A. Sanguesa, M. Fogue, P. Garrido, F. J. Martinez, J.-C. Cano, and C. T. Calafate, "Topology-based broadcast schemes for urban scenarios targeting adverse density conditions," in Proceedings of the IEEE Wireless Communications and Networking Conference (WCNC '14), pp. 2528-2533, IEEE, Istanbul, Turkey, April 2014.

[52] J. A. Sanguesa, M. Fogue, P. Garrido, F. J. Martinez, J.-C. Cano, and C. T. Calafate, "Using topology and neighbor information to overcome adverse vehicle density conditions," Transportation Research Part C: Emerging Technologies, vol. 42, pp. 1-13, 2014.

[53] S.-I. Sou and Y. Lee, "SCB: store-carry-broadcast scheme for message dissemination in sparse VANET," in Proceedings of the IEEE 75th Vehicular Technology Conference (VTC Spring '12), pp. 1-5, IEEE, Yokohama, Japan, May 2012.

[54] O. K. Tonguz, N. Wisitpongphan, and F. Bai, "DV-CAST: a distributed vehicular broadcast protocol for vehicular ad hoc networks," IEEE Wireless Communications, vol. 17, no. 2, pp. 47$57,2010$.

[55] W. Viriyasitavat, O. K. Tonguz, and F. Bai, "UV-CAST: an urban vehicular broadcast protocol," IEEE Communications Magazine, vol. 49, no. 11, pp. 116-124, 2011.

[56] D. Sormani, G. Turconi, P. Costa, D. Frey, M. Migliavacca, and L. Mottola, "Towards lightweight information dissemination in inter-vehicular networks," in Proceedings of the 3 rd International Workshop on Vehicular Ad Hoc Networks (VANET '06), pp. 2029, ACM, Los Angeles, Calif, USA, September 2006.

[57] J. A. Sanguesa, M. Fogue, P. Garrido et al., "RTAD: a realtime adaptive dissemination system for VANETs," Computer Communications, vol. 60, pp. 53-70, 2015.

[58] TIGER, "Topologically Integrated Geographic Encoding and Referencing," 2015, http://www.census.gov/geo/www/tiger.

[59] OpenStreetMap, "Collaborative project to create a free editable map of the world," 2015, http://www.openstreetmap.org.

[60] F. J. Martinez, C.-K. Toh, J.-C. Cano, C. T. Calafate, and P. Manzoni, "Realistic radio propagation models (RPMs) for VANET simulations," in Proceedings of the IEEE Wireless Communications and Networking Conference (WCNC '09), pp. 1-6, IEEE, Budapest, Hungary, April 2009.

[61] H. T. Friis, "A note on a simple transmission formula," Proceedings of the IRE, vol. 34, no. 5, pp. 254-256, 1946.

[62] T. S. Rappaport, Wireless Communications: Principles and Practice, Prentice Hall-PTR, Upper Saddle River, NJ, USA, 2nd edition, 2001.

[63] W. Viriyasitavat, F. Bai, and O. K. Tonguz, "Dynamics of network connectivity in urban vehicular networks," IEEE Journal on Selected Areas in Communications, vol. 29, no. 3, pp. 515-533, 2011.

[64] F. J. Martinez, M. Fogue, C. K. Toh, J.-C. Cano, C. T. Calafate, and P. Manzoni, "Computer simulations of VANETs using realistic city topologies," Wireless Personal Communications, vol. 69, no. 2, pp. 639-663, 2013.

[65] W. Alasmary and W. Zhuang, "Mobility impact in IEEE 802.11p infrastructureless vehicular networks," Ad Hoc Networks, vol. 10, no. 2, pp. 222-230, 2012.

[66] C.-K. Toh, Ad Hoc Mobile Wireless Networks: Protocols and Systems, Prentice Hall, Upper Saddle River, NJ, USA, 2001.

[67] D. Cavin, Y. Sasson, and A. Schiper, "On the accuracy of MANET simulators," in Proceedings of the 2nd ACM International Workshop on Principles of Mobile Computing (POMC '02), pp. 38-43, ACM, Toulouse, France, October 2002.

[68] J. Yoon, M. Liu, and B. Noble, "Random waypoint considered harmful," in Proceedings of the Twenty-Second Annual Joint Conference of the IEEE Computer and Communications (INFOCOM '03), vol. 2, pp. 1312-1321, San Francisco, Calif, USA, MarchApril 2003.

[69] T. Camp, J. Boleng, and V. Davies, "A survey of mobility models for ad hoc network research," Wireless Communications \& Mobile Computing, vol. 2, no. 5, pp. 483-502, 2002. 
[70] S. Krauss, P. Wagner, and C. Gawron, "Metastable states in a microscopic model of traffic flow," Physical Review E, vol. 55, no. 5, pp. 5597-5602, 1997.

[71] O. K. Tonguz, W. Viriyasitavat, and F. Bai, "Modeling urban traffic: a cellular automata approach," IEEE Communications Magazine, vol. 47, no. 5, pp. 142-150, 2009.

[72] K. Fall and K. Varadhan, "ns notes and documents," The VINT Project. UC Berkeley, LBL, USC/ISI, and Xerox PARC, February 2000, http://www.isi.edu/nsnam/ns/ns-documentation.html.

[73] Riverbed, “OPNET Modeler Suite," 2015, http://www.opnet .com/.

[74] Real-Time \& Embedded Systems Lab, "GrooveneNet, a vehicular network virtualization platform," 2012, http://mlab.seas.upenn.edu/groovenet/.

[75] M. Fogue, P. Garrido, F. J. Martinez, J.-C. Cano, C. T. Calafate, and P. Manzoni, "A realistic simulation framework for vehicular networks," in Proceedings of the 5th International ICST Conference on Simulation Tools and Techniques (SIMUTools '12), pp. 37-46, Desenzano del Garda, Italy, March 2012.

[76] M. Fogue, P. Garrido, F. J. Martinez, J.-C. Cano, C. T. Calafate, and P. Manzoni, "Identifying the key factors affecting warning message dissemination in VANET real urban scenarios," Sensors, vol. 13, no. 4, pp. 5220-5250, 2013.

[77] S. Ucar, S. Coleri Ergen, and O. Ozkasap, "Multi-hop cluster based IEEE 802.11p and LTE hybrid architecture for VANET safety message dissemination," IEEE Transactions on Vehicular Technology, 2015. 

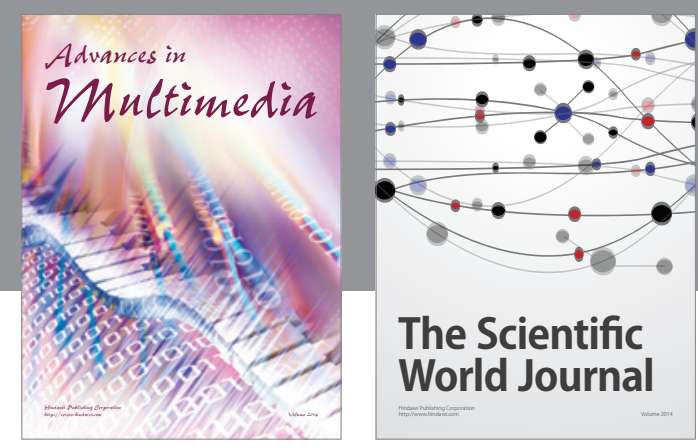

The Scientific World Journal
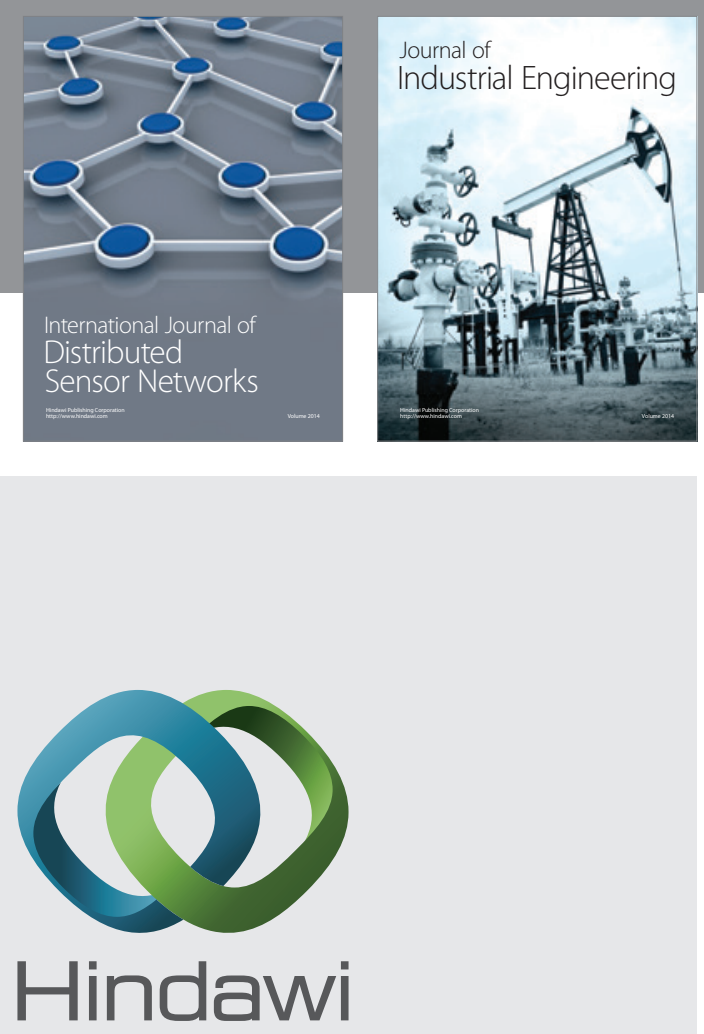

Submit your manuscripts at

http://www.hindawi.com

\section{Computer Networks} and Communications
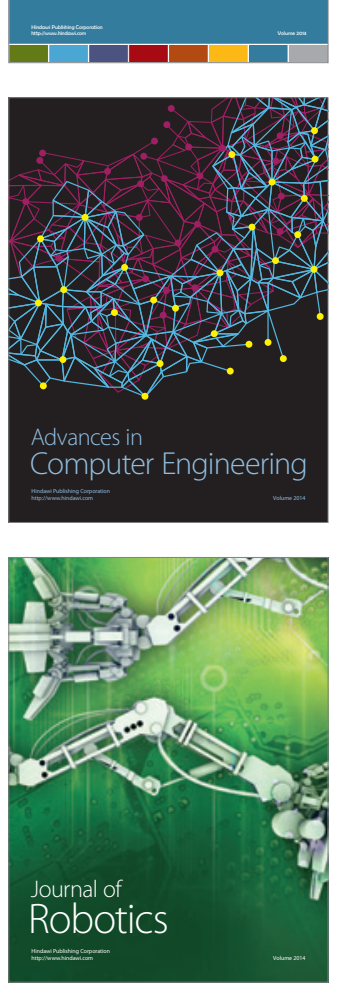
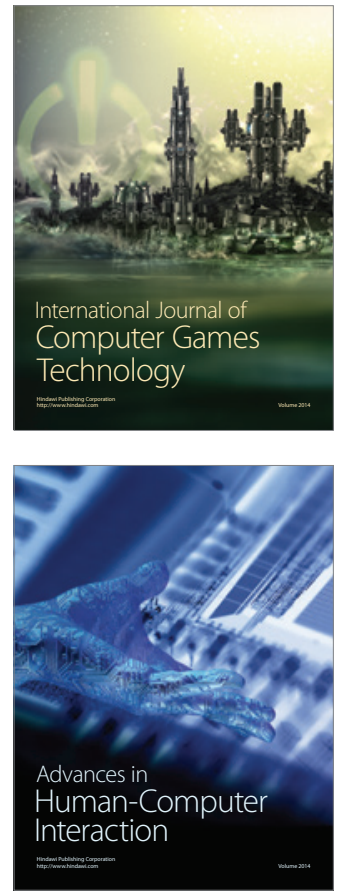
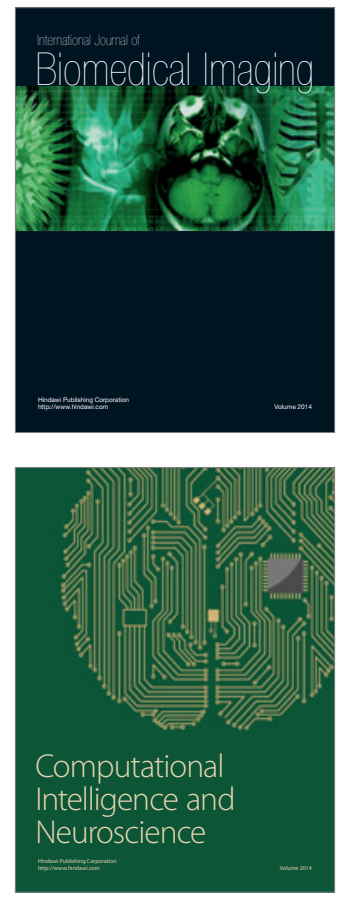
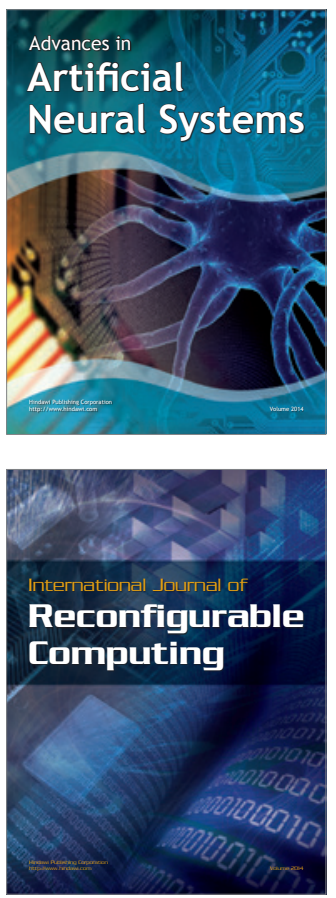
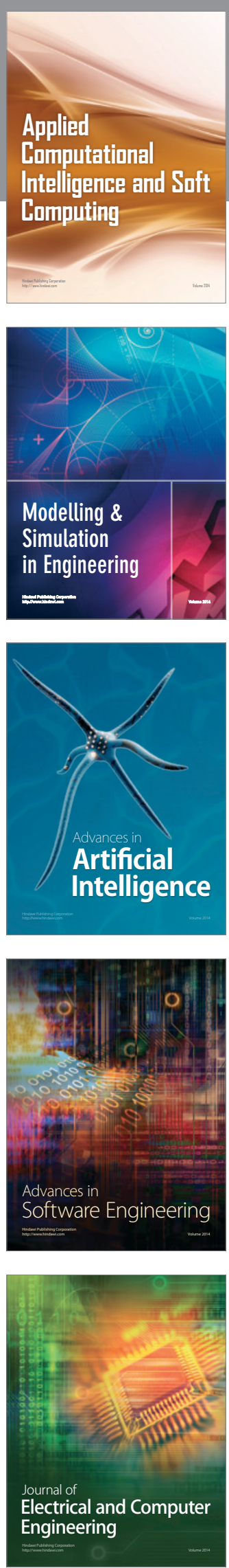